\title{
One-loop electromagnetic correlators of SQED in power-law inflation
}

\author{
Dražen Glavan, ${ }^{1}$ Gerasimos Rigopoulos ${ }^{2}$ \\ ${ }^{1}$ Centre for Cosmology, Particle Physics and Phenomenology (CP3), \\ Université catholique de Louvain, \\ Chemin du Cyclotron 2, 1348 Louvain-la-Neuve, Belgium \\ ${ }^{2}$ School of Mathematics, Statistics and Physics, \\ Herschel Building, Newcastle University, \\ Newcastle upon Tyne, NE1 7RU, United Kingdom \\ E-mail: drazen.glavan@uclouvain.be, \\ gerasimos.rigopoulos@newcastle.ac.uk
}

\begin{abstract}
:
We examine scalar quantum electrodynamics in power-law inflation, and compute the one-loop correction to electric and magnetic field correlators at superhorizon separations. The effect at one-loop descends from the coupling of the vector to the charged scalar current which is greatly enhanced due to gravitational particle production. We conclude that non-perturbative effects must exist due to (i) secular growth, (ii) spatial running, and (iii) infrared sensitivity of the one-loop correction to the correlators. Electric and magnetic correlators exhibit a hierarchy that is due to Faraday's law and accelerated expansion, and must hold non-perturbatively.
\end{abstract}




\section{Introduction}

The idea of utilizing the primordial inflationary universe as a testing ground for Beyond Standard Model (BSM) particle physics - dubbed the Cosmological Collider - has gained prominence lately [1,2]. The high energy scale of the expansion in primordial inflation can excite very heavy matter fields inaccessible to foreseeable Earth-base accelerators. These, in turn, could have left an imprint on cosmological observables. However, if we are to sift through the signals from primordial inflation for signs of BSM physics, it is paramount that we first understand the behaviour of the Standard Model (SM) itself in the extreme gravitational conditions of the rapidly expanding and accelerating Universe.

The SM fields are all light in inflation, relative to the Hubble scale, $H \sim 10^{13} \mathrm{GeV}$, which can lead to drastically different phenomenology compared to flat space. In particular, the fields that couple non-conformally to gravity - light scalars and the graviton - are influenced by the expansion the most. Even at tree-level they experience the so-called gravitational particle production [3-7]. The virtual pairs of particles, popping in and out of existence due to quantum fluctuations, are stretched to superhorizon separations by the rapid expansion before they can annihilate, and thus the expansion rips them from the vacuum into existence, as depicted schematically in Fig. 1. This infrared (IR) effect is so strong that in general it leads to the symmetry restoration of symmetry breaking potentials in scalar models [8-12]. The Higgs field of the SM is extremely light in inflation, so it experiences large IR effects.

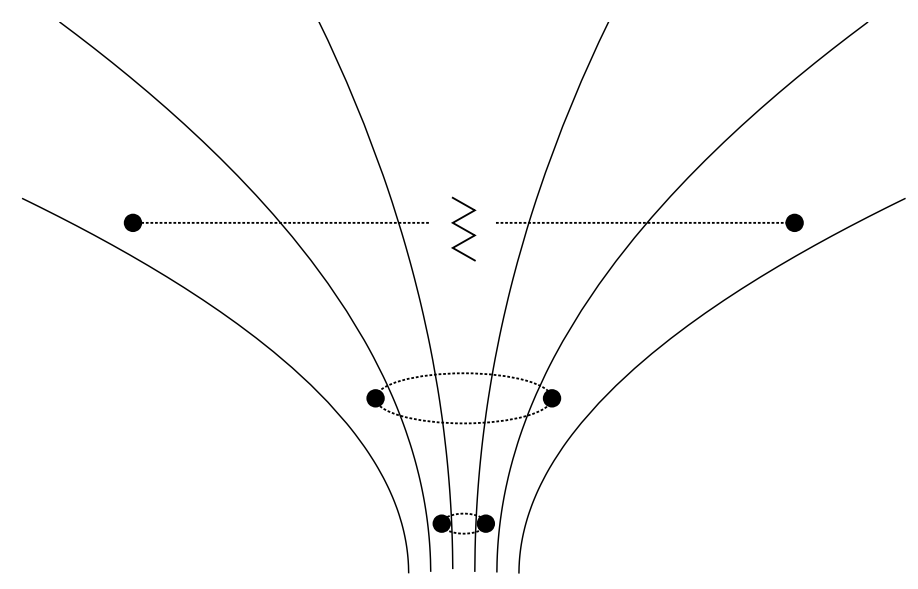

Figure 1. Gravitational particle production. A virtual pair created as a quantum fluctuation is stretch rapidly by the expanding space to the point where its lifetime becomes infinite, and it is ripped out of the vacuum into existence.
On the other hand, fields that couple conformally to gravity, such as gauge bosons or fermions, do not experience gravitational particle productions in conformally flat cosmological space-times, as they effectively do not distinguish between the expanding space and flat space. ${ }^{1}$ Nevertheless, couplings of those fields to the graviton, or a light scalar such as the SM Higgs field, can communicate to them the effects of the expansion. Therefore, it is to be expected that all SM fields

\footnotetext{
${ }^{1}$ Quantum effects generally make the conformal symmetry anomalous [13], which breaks conformality only mildly (see also [14] for the implications on primordial magnetogenesis).
} 
can be influenced by the expansion of primordial inflation, and it is important to understand what kind of signals they could impart on the cosmological observables. This question was emphasized recently in [15-17], where the authors concentrated on how inflation changes the mass spectrum of the SM. However, as we show here, mass corrections might not be the only signal of the SM in inflation.

This work is primarily motivated by the question of how do the boson fields of the SM electroweak (EW) sector behave in primordial inflation, and in particular how are they affected by loop corrections from the Higgs field with large IR enhancement. To this end we study a simplified model - scalar quantum electrodynamics (SQED) - that contains a light complex scalar with $U(1)$ charge, and a vector that couples to it. Since the complex scalar is charged, gravitational particle production now creates pairs of opposite charge. The vector field couples to the created charges and the currents

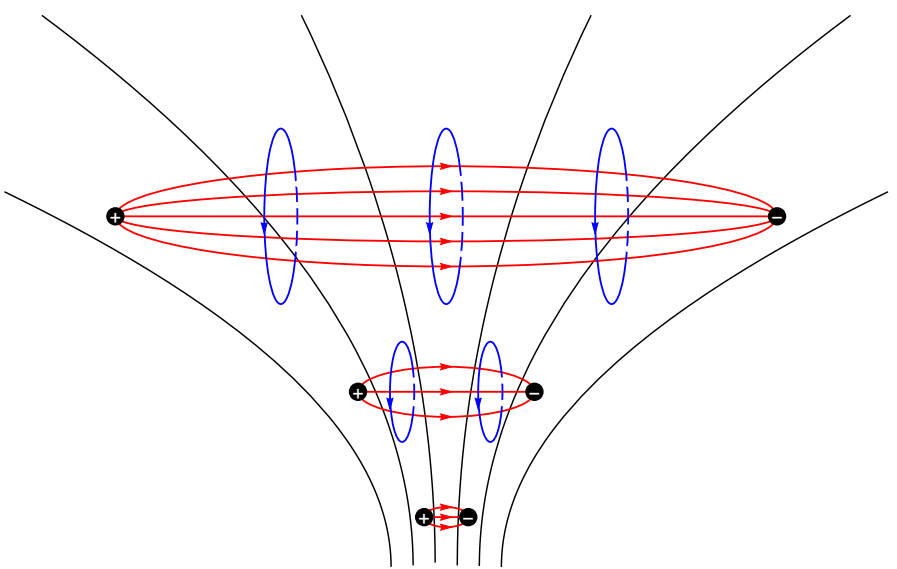

Figure 2. Gravitational particle production of charged particles. Particles of the pair created by gravitational particle production carry opposite charges, and couple to the photon field. The photon field reacts to created charges resulting in electric (red) and magnetic (blue) fields. The created electric and magnetic fields further act on the charges, and may lead to further creation of charged pairs via Schwinger mechanism. they produce, establishing electric and magnetic (E\&M) fields. As the charged pair is stretched to superhorizon separations, so are the E\&M fields, as is schematically depicted in Fig. 2.

The reaction of the vector field to gravitationally induced charged currents, and the resulting superhorizon electric and magnetic fields, are one-loop quantum effects. In this work we compute the one-loop correction to the superhorizon correlators of quantum fluctutions of E\&M fields,

$$
\left\langle\hat{F}_{\mu \nu}(\eta, \vec{x}) \hat{F}_{\rho \sigma}\left(\eta, \vec{x}^{\prime}\right)\right\rangle,
$$

for SQED in power-law inflation. The equal-time E\&M field correlators, induced here due to interacting quantum fluctuations, bear observational relevance in relation to the problem of cosmic magnetic fields, ${ }^{2}$ and their primordial origin [21-24]. The power-law inflation, characterized by a constant non-zero slow-roll parameter, $\epsilon=$

\footnotetext{
${ }^{2}$ See $[18-20]$ for recent reviews.
} 
$-\dot{H} / H^{2}$, provides a better approximation for the more realistic slow-roll inflation, compared to the de Sitter space, $\epsilon=0$, in which most previous works are set. This is crucial for secular effects coming from quantum loops, whose scaling generally depends on $\epsilon$.

Investigations of the quantum loop effects of SQED in inflation originated in $[25$, 26], where it was argued that the vector field develops an effective mass due to the large IR fluctuations of the complex scalar. This argument was based on the Hartree approximation descending from the 4-point interaction, which resembles a photon mass. It was inferred that the generation of such a mass has potential observational significance in creating cosmological magnetic fields. Even though the Hartree approximation is known not to be reliable quantitatively, the conjecture of the photon mass generation in massless SQED in de Sitter was nevertheless subsequently confirmed for a photon propagating through an ensemble of superhorizon quantum fluctuations in [27-29], where the full one-loop computation of the vacuum polarization was performed. However, unlike the original suggestion of [25, 26], the origin of the photon mass turned out not to be the 4-point interaction, but rather the 3-point one. ${ }^{3}$ The mass of the photon was inferred by examining the one-loop correction to the photon mode function, and it was found that the sub-Hubble dynamics is captured by a Proca-like photon mass term, $m_{\gamma}^{2}=\frac{(q H)^{2}}{2 \pi^{2}} \times \ln (k / H)$, where $k$ is the modulus of the photon's wave-vector, and $H$ is the de Sitter space Hubble rate, and $q$ is the charge of the scalar. Furthermore, in [30] the one-loop corrected equations for electric and magnetic fields were solved self-consistently, and it was reported that the electric field of the long-wavelength photon receives power-law secular enhancement $\sim a^{1 / 2}$, while the magnetic field gets a constant amplitude renormalization. The conclusion was that the dynamics of the photon was more intricate than it just developing a local effective mass. The investigation was extended to light massive and non-minimally coupled SQED in [31,32], with similar conclusions, the generated photon mass being $m_{\gamma}^{2}=\frac{(q H)^{2}}{2 \pi^{2}} \times 3 H^{2} /\left[2\left(m_{\phi}^{2}+12 \xi H^{2}\right)\right]$, where $m_{\phi}$ is the complex scalar's mass, and $\xi$ is the dimensionless non-minimal coupling (see also [33]). Again, the effect in the massive case also derives only from the 3-point interaction.

Investigations of SQED in de Sitter were greatly advanced in the seminal work [34], where the authors adapted Starobinsky's stochastic formalism [35] to SQED in de Sitter. This was accomplished in two steps: (i) the vector field was integrated out exactly, owing to it appearing only quadratically in the action, and the effective non-local scalar theory was obtained, and (ii) Starobinsky's stochastic equation was derived for the gauge-invariant scalar field variance $\Phi^{*} \Phi$ from the effective scalar theory, and solved in the late-time limit. Thus, the leading order IR effects on

\footnotetext{
${ }^{3}$ The contribution of the 4-point interaction to the vacuum polarization is precisely canceled by the local part of the 3-point contribution. The only local term surviving is the conformal anomaly.
} 
the scalar have been resummed ${ }^{4}$ to reveal that it develops a non-Gaussian interacting state with a non-perturbatively large variance, $\left\langle\Phi^{*} \Phi\right\rangle \approx 1.65 \times H^{2} / q^{2}$, but a perturbatively small mass $m_{\phi}^{2} \approx 0.45 \times(q H)^{2} /(2 \pi)^{2}$. This implies that a photon propagating through such a polarizable medium develops a non-perturbatively large effective mass, $m_{\gamma}^{2}=2 q^{2}\left\langle\Phi^{*} \Phi\right\rangle \approx 3.3 \times H^{2}$. The work on the stochastic formalism was followed up by two-loop computations of the local scalar bi-linears, and the energy-momentum tensor, confirming its consistency [38, 39].

Recently, comprehensive computations of the one-loop corrections to two-point functions of SM fields in de Sitter were reported in [15-17]. Out of the many relevant cases that the authors considered, here we comment only on those pertinent to our work - loop corrections to the 2-point function of vector bosons in SQED and the EW sector of the SM. Two diagrams contribute at one-loop to the vector boson propagator correction, a local one constructed from a 4-vertex, and a non-local one made up of 3-vertices, and both contributions are reported to exhibit secular growth, which is in accord with other perturbative results. The correction descending from the local diagram is recognized to have the form of a mass insertion, which in [15-17] is resummed using the ansatz of the Dynamical Renormalization Group (DRG) [40, 41]. Thus, the authors conclude that the effect the expanding space-time and the coupling to a light scalar have on vector fields is to change their mass spectrum. However, it should be noted that the contribution of the local one-loop diagram cancels out when all one-loop corrections are accounted for [27, 31], and that it is actually the non-local diagram, constructed out of 3 -vertices, that provides the leading correction. Furthermore, the DRG is known to treat the local diagrams correctly, reproducing the known results for the scalar fields [42-46], but not for the non-local diagrams relevant for vector fields. Even though, interestingly, the reported one-loop generated mass [15] of the vector in SQED matches the one from [31], these have a completely different origin. Moreover, adopting the unitary gauge to infer the loop corrections to the EW sector of the SM in the symmetric phase seems problematic, since the same arguments applied to SQED would eliminate the 3-vertices that are responsible for the dominant one-loop contribution.

The computation of the one-loop correction to the equal-time E\&M correlators that we present here differs from the previous works in the following aspects:

- We compute the full two-point function of the E\&M fields in order to quantify the loop correction, instead of the vector field mode function computed in $[27$, 39]. While it is true that the one-loop corrected mode function contains the same information as the one-loop corrected two-point function, and one can be reconstructed from another, they have different physical meaning. The photon mode function gives the information about the photon that propagates through

\footnotetext{
${ }^{4}$ For one-loop corrections the photon induces on the dynamics of the scalar see [36, 37].
} 
a polarizable medium, the correlators of E\&M fields provide information about the polarizable medium itself. This is the kind of information closer to the cosmological observables such as the cosmic microwave background that comes in the form of correlators across the sky.

- We compute a two-point function of a gauge-invariant observable, rather than the gauge-dependent two-point function of the vector field as in [15-17]. While there is nothing wrong with the latter, the physical interpretation is more obscure due to gauge dependence. This is particularly delicate when it comes to recognizing generated dynamical effective mass of a vector field, which cannot readily be inferred by comparing it to the Proca field.

- Effects at superhorizon separations that we consider descend from the non-local one-loop diagram composed out of 3-vertices of SQED, unlike Refs. [15-17] that quote the local one-loop diagram, containing only one 4-vertex, as the origin of the photon mass generation. Our work is in line with Refs. [27, 31] which demonstrated that the only local effect that does not cancel is in fact just the conformal anomaly.

- We consider the background to be power-law inflation, characterized by a finite and constant slow-roll parameter $0<\epsilon<1$, as opposed to the exact de Sitter space-time $(\epsilon=0)$ considered previously. Observations suggest that primordial inflation had a finite and slowly-changing slow-roll parameter of the order $\epsilon \sim$ 0.01 , as measured by the spectral tilt of primordial fluctuations. Power-law inflation does not represent this faithfully, but it does capture the effects of the finite slow-roll parameter. Even though the de Sitter approximation is often good, but for the purposes of loop corrections in inflation it does not have to be so, as they typically exhibit secular corrections. A secular correction that scales as $\sim \ln (a)$ in de Sitter might exhibit scaling of the form $\sim\left(a^{\epsilon}-1\right) / \epsilon$ in power-law inflation. It is not really known how long inflation might have lasted, and given enough time these two scalings start deviating considerably. This expectation is corroborated by the computation presented here. Moreover, working in power-law inflation as opposed to exact de Sitter allows us to break the degeneracy between the (effective) particle mass and the Hubble rate. Some works would suggest that fields tend to develop effective non-minimal coupling to the Ricci scalar, as opposed to the constant mass term [47, 48]. The two have different scalings, and it is important to distinguish between the two.

The computation of the one-loop corrections that we report here is not performed by evaluating the integrals over two-point functions corresponding to one-loop Feyn- 
man diagrams given below in Figs. 4 and $5 .{ }^{5}$ Instead, we formulate the problem in terms of double-differential equations descending solely from forming correlators of operator Maxwell's equations with themselves, reminiscent of Dyson-Schwinger equations in the Schwinger-Keldysh formalism $[49,50]$. Solving the equations directly in the superhorizon limit is straightforward, and yields the one-loop correction to the superhorizon correlators. A similar approach has already been employed in [22-24] when computing magnetic field correlators in the radiation era (see also [51] for recent work on the topic). This procedure greatly simplifies the technical difficulties of loop computations in inflation, if at the end of the day one is interested only in superhorizon behaviour, and potentially makes the two-loop computation feasible.

We find that all the one-loop E\&M correlators are enhanced compared to the tree-level vacuum fluctuations. The enhancement is manifested in three aspects: (i) secular growth, (ii) spatial running, and (iii) IR sensitivity. We find that in powerlaw inflation the secular growth of E\&M correlators is more pronounced than in de Sitter space, thus making the perturbation theory even less well behaved. However, it is unclear exactly whether, or which aspect of the result ought to be considered as an artefact of time-dependent perturbation theory. Superhorizon correlators also grow with comoving spatial separation, providing yet another limitation of perturbation theory. Finally, The IR sensitivity is manifested by the almost logarithmic dependence of the correlators on the deep IR scale of the initial state, necessary to define the physical state of the complex scalar. All three enhancements signal potentially large non-perturbative effects for the E\&M correlators of massless SQED in power-law inflation, and further investigation is clearly called for.

Even though the magnitude of non-perturbative effects is beyond the scope of this work, the one-loop computation reported here does offer an important insight. The correlators that we compute, both for the conserved $U(1)$ current and for the E\&M fields, satisfy distinct hierarchies. For current correlators the hierarchy between components is $(i j) \gg(0 i) \gg(00)$, while the E\&M correlators the hierarchy is $(E E) \gg$ $(E B) \gg(B B)$. Both of the hierarchies are in fact double hierarchies, satisfied by both the secular growth, and by the spatial running. These hierarchies depend only on the homogeneous operator equations: the conservation equation for the current and Faraday's law of induction for the E\&M fields, and they have to hold generally in inflating space-times even at the non-perturbative level. In a decelerating universe after inflation Faraday's law [52] work towards inverting this hierarchy, leading to transient effects reported in [52]. The amplitude of the correlators, on the other hand, is not universal and depends on the specificities of the model and the background space-time. The linear fluctuations in the symmetry-breaking Abelian Higgs model in power-law inflation considered recently in [48] demonstrates explicitly the hierarchy

\footnotetext{
${ }^{5}$ This would in fact not be possible as the photon propagators for power-law inflation have not been worked out thus far.
} 
reported here.

The remainder of the paper is organized as follows: The following section introduces the power-law inflation space-time, and the SQED model in it. Sec. 3 discusses our approach to quantifying quantum fluctuations - computing correlators of charge currents and EM fields, while Sec. 4 and 5, are devoted to computing the said correlators. The general hierarchies between the superhorizon correlators are discussed in Sec. 6, while the last section is reserved for the discussion of the results.

\section{Scalar electrodynamics in FLRW}

In this section we briefly introduce the power-law inflation space-time and the scalar quantum electrodynamics model living in it.

\subsection{FLRW and power-law inflation}

The invariant line element of spatially flat Friedmann-Lemaître-Robertson-Walker (FLRW) space-time in conformal coordinates is given by,

$$
d s^{2}=a^{2}(\eta)\left[-d \eta^{2}+d \vec{x}^{2}\right]
$$

where $a(\eta)$ is the scale factor expressed in terms of conformal time $\eta$, and hence the metric is conformally flat, $g_{\mu \nu}=a^{2} \eta_{\mu \nu}$, where the Minkowski metric is $\eta_{\mu \nu}=$ $\operatorname{diag}(-1,1,1,1)$. A quantity which encodes the rate of change of the scale factor is the conformal Hubble rate,

$$
\mathcal{H}=\frac{\partial_{0} a}{a},
$$

which is related to the physical Hubble rate $H$ as $\mathcal{H}=a H$. Acceleration of the expansion is conveniently encoded by the so-called principal slow-roll parameter,

$$
\epsilon \equiv 1-\frac{\partial_{0} \mathcal{H}}{\mathcal{H}^{2}}
$$

related to the often used deceleration parameter $q$ as $\epsilon=q+1$. When $\epsilon<1$ the expansion is accelerating, and when $\epsilon>1$ it is decelerating. In primordial inflation it typically takes small values $0<\epsilon \ll 1$. In this work we consider the special class of FLRW space-times - power-law inflation - characterized by the constant principal slow-roll parameter,

$$
\epsilon=\text { const } \quad 0 \leq \epsilon<1
$$

The scale factor and the conformal Hubble rate in power-law inflation are related as $\mathcal{H}=H_{0} a^{1-\epsilon}$, and have particular time dependencies,

$$
a(\eta)=\left[1-(1-\epsilon) H_{0}\left(\eta-\eta_{0}\right)\right]^{\frac{-1}{1-\epsilon}}, \quad \mathcal{H}(\eta)=H_{0}\left[1-(1-\epsilon) H_{0}\left(\eta-\eta_{0}\right)\right]^{-1},
$$


where $a\left(\eta_{0}\right)=1$ and $\mathcal{H}\left(\eta_{0}\right)=H_{0}$. The ranges of coordinates in power-law inflation are,

$$
-\infty<\eta<\bar{\eta} \equiv \eta_{0}+\frac{1}{(1-\epsilon) H_{0}}, \quad-\infty<x^{i}<\infty
$$

and the conformal diagram of the space-time is given in Fig. 3. In this paper we shall

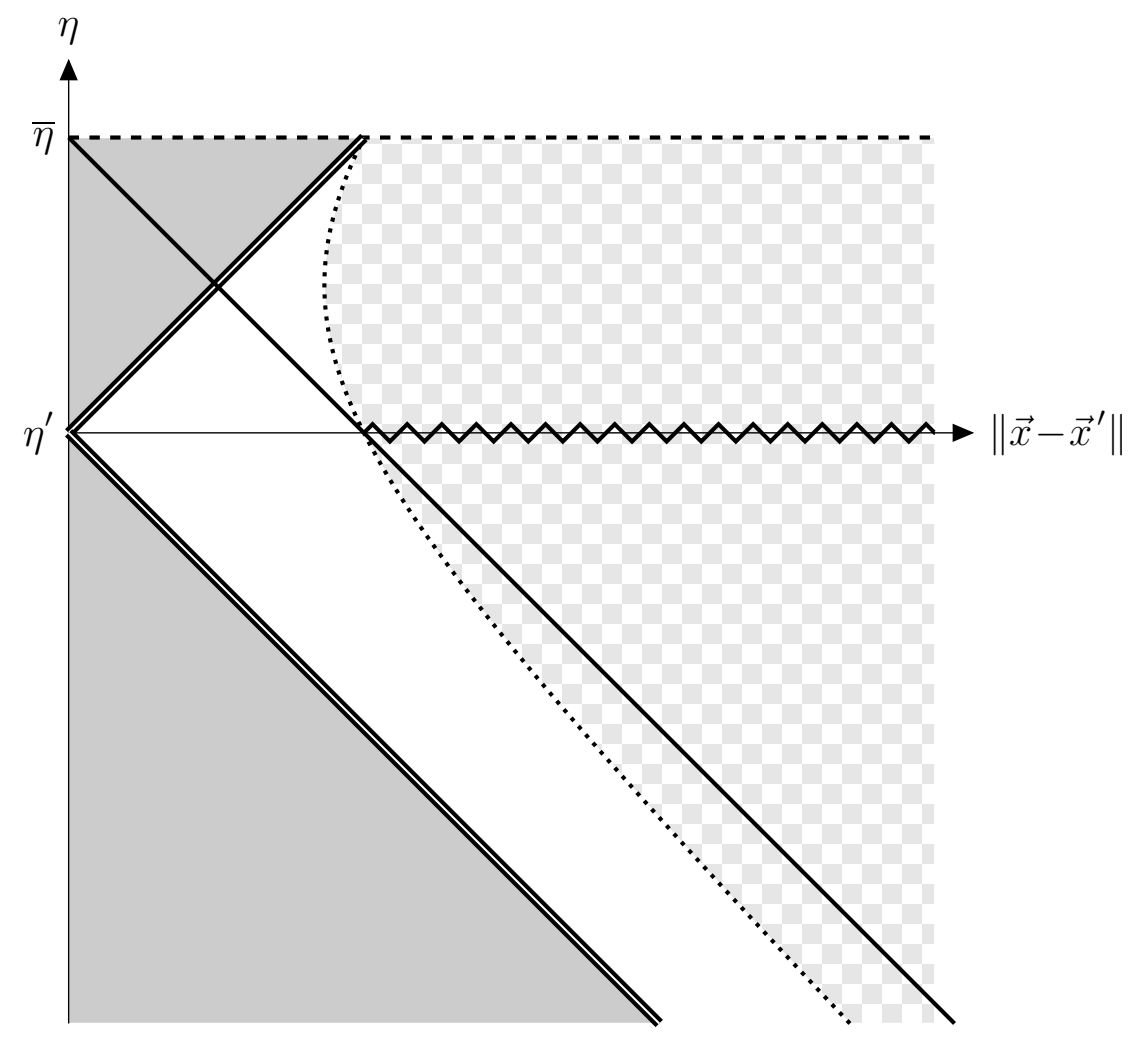

Figure 3. Conformal diagram of power-law inflation space-time. Two double lines represent the past and the future null rays of some point $\left(\eta^{\prime}, \vec{x}^{\prime}\right)$. The gray shaded region represents its past and future light-cones, and from the allowed range of coordinates in Eq. (2.6), it follows that the past light-cone can grow without limits, while the future light cone is limited by the asymptotic future time $\bar{\eta}$. The dotted curve along with the checkered region represent generalized superhorizon separations $(\overline{\mathrm{SH}})$ from Eq. (2.8), while the jagged (zig-zag) line represents the spatial points $\left(\eta^{\prime}, \vec{x}\right)$ at superhorizon (SH) separations from $\left(\eta^{\prime}, \vec{x}^{\prime}\right)$. The bold solid line denotes the Hubble horizon of the comoving observer at $\vec{x}^{\prime}$.

consider a more restricted class of power-law inflation for which $0<\epsilon<1 / 2$ for the sake of simplicity, which is also appropriate for inflation applications where $0<\epsilon \ll 1$.

In this work we mostly deal with correlators - bi-local objects dependent on two points of the manifold which we denote by $x$ and $x^{\prime}$. Henceforth, in bi-local objects, primed quantities are assumed dependent on the primed coordinate $x^{\prime}$, and while unprimed quantities are assumed dependent on the unprimed coordinate $x$, 
e.g. $\mathcal{H}^{\prime}=\mathcal{H}\left(\eta^{\prime}\right)$, and $\mathcal{H}=\mathcal{H}(\eta)$. It is convenient for us to define the standard notion of superhorizon separation between two points $x$ and $x^{\prime}$ at equal time slices,

$$
\mathrm{SH}: \quad\|\Delta \vec{x}\| \gg \frac{1}{(1-\epsilon) \mathcal{H}}, \quad \eta^{\prime}=\eta,
$$

and also the notion of generalized superhorizon separation for non-equal times,

$$
\overline{\mathrm{SH}}: \quad\|\Delta \vec{x}\|^{2}-\left(\eta-\eta^{\prime}\right)^{2} \gg \frac{1}{(1-\epsilon)^{2} \mathcal{H} \mathcal{H}^{\prime}},
$$

where $\Delta \vec{x}=\vec{x}-\vec{x}^{\prime}$. The latter is introduced to allow us to take time derivatives of bi-local functions in intermediate steps of the computations, but at the end we are interested in the time-coincidence limit. The symbols $\stackrel{\mathrm{SH}}{\sim}$ and $\stackrel{\overline{\mathrm{SH}}}{\sim}$ will denote the two limits, respectively.

\subsection{SQED in FLRW}

The action for scalar electrodynamics in a 4-dimensional curved space-time is given by,

$$
S\left[A_{\mu}, \Phi, \Phi^{*}\right]=\int d^{4} x \sqrt{-g}\left[-\frac{1}{4} g^{\mu \rho} g^{\nu \sigma} F_{\mu \nu} F_{\rho \sigma}-g^{\mu \nu}\left(D_{\mu}^{*} \Phi^{*}\right)\left(D_{\nu} \Phi\right)\right],
$$

where $F_{\mu \nu}=\partial_{\mu} A_{\nu}-\partial_{\nu} A_{\mu}$ is the vector field strength tensor, $D_{\mu}=\nabla_{\mu}+i q A_{\mu}$ is the $U(1)$ covariant derivative, $q$ is the coupling constant (the $U(1)$ charge), and $g=\operatorname{det}\left(g_{\mu \nu}\right)$ is the metric determinant. The equations of motion for the classical theory descending from this action are,

$$
g^{\rho \sigma} \nabla_{\rho} F_{\sigma \mu}=q J_{\mu}, \quad \quad g^{\mu \nu} D_{\mu} D_{\nu} \Phi=0,
$$

where the source for the Maxwell's equation above is the $U(1)$ Noether's current,

$$
J_{\mu}=i\left[\left(D_{\mu}^{*} \Phi^{*}\right) \Phi-\Phi^{*}\left(D_{\mu} \Phi\right)\right]=i\left[\left(\partial_{\mu} \Phi^{*}\right) \Phi-\Phi^{*}\left(\partial_{\mu} \Phi\right)\right]+2 q A_{\mu} \Phi^{*} \Phi,
$$

which is covariantly conserved,

$$
g^{\mu \nu} \nabla_{\mu} J_{\nu}=0
$$

as a consequence of the second (scalar field) equation. Note that $\Phi^{*}$ satisfies the complex conjugate of the second equation in (2.10). In addition to dynamical equations in (2.10), the vector field strength also satisfies homogeneous Maxwell's equations simply on the account of its anti-symmetry, $\nabla_{[\mu} F_{\nu \rho]}=0$.

The dynamics of the quantum theory (scalar quantum electrodynamics - SQED) is captured by the equations of motion formally of the same form as the classical equations (2.10), where the fields are substituted by the field operators,

$$
A_{\mu} \rightarrow \hat{A}_{\mu}, \quad \Phi \rightarrow \hat{\Phi}, \quad \Phi^{*} \rightarrow \hat{\Phi}^{\dagger}
$$


everywhere in the equations, including the covariant derivatives, and the conserved current in (2.11). In addition, the field operators in (2.13) satisfy canonical commutation relations, which for gauge theories such as SQED depend on the choice of gauge. Even though our results are gauge-invariant, for definiteness we will be working in the exact covariant gauge, ${ }^{6}$

$$
g^{\mu \nu} \nabla_{\mu} \hat{A}_{\nu}=0
$$

which is implicit in the operator version of equations of motion (2.10).

\section{Quantum fluctuations}

We are interested in computing the properties of electric and magnetic fields in a globally (and locally) neutral state, that contains no condensates of electric and magnetic fields,

$$
\left\langle\hat{J}_{\mu}(x)\right\rangle=0, \quad\left\langle\hat{F}_{\mu \nu}(x)\right\rangle=0 .
$$

Even though the state has no net charge, because of the fundamental quantum nature of the fields they do exhibit quantum fluctuations, i.e. correlators of currents and field strengths do not vanish,

$$
\left\langle\hat{J}_{\mu}(x) \hat{J}_{\nu}\left(x^{\prime}\right)\right\rangle \neq 0, \quad\left\langle\hat{F}_{\mu \nu}(x) \hat{F}_{\rho \sigma}\left(x^{\prime}\right)\right\rangle \neq 0 .
$$

These correlators are what we wish to compute in time coincidence and for superhorizon distances, as defined in (2.7). In addition to being physically relevant, the superhorizon limit allows us to simplify the computation considerably. Instead of going through the standard diagrammatic approach to one-loop computations, we choose to present the computation in a more intuitive framework that ties better to way things are thought of in cosmology.

The tree-level correlator of vector field strength, in absence of coupling to charged fields in power-law inflation (and in general FLRW) is given by

$$
\left\langle\hat{F}_{\mu \nu}(x) \hat{F}_{\rho \sigma}\left(x^{\prime}\right)\right\rangle=\frac{2}{\pi^{2}\left(\Delta x^{2}\right)^{2}}\left[\eta_{\mu[\rho} \eta_{\sigma] \nu}-4 \eta_{\alpha[\mu} \eta_{\nu][\sigma} \eta_{\rho] \beta} \frac{\Delta x^{\alpha} \Delta x^{\beta}}{\Delta x^{2}}\right],
$$

where $\Delta x^{\mu}=x^{\mu}-x^{\prime \mu}$, and $\Delta x^{2}=\eta_{\mu \nu} \Delta x^{\mu} \Delta x^{\nu}$. This correlator takes the same form as in flat space due to: (i) vector field being conformally coupled to gravity in $D=4$, (ii)

\footnotetext{
${ }^{6}$ This gauge condition should be thought of as the $\xi \rightarrow 0$ limit of the general covariant averaged gauge, given by the gauge-fixing action,

$$
S_{\mathrm{gf}}\left[A_{\mu}\right]=\int d^{4} x \sqrt{-g}\left[-\frac{1}{2 \xi}\left(g^{\mu \nu} \nabla_{\mu} A_{\nu}\right)^{2}\right],
$$

which is appropriate for the indefinite metric quantization.
} 
FLRW space-time being conformally flat, and (iii) defining a conformally invariant state for the vector field.

The vector field in SQED is sourced by the complex scalar via the $U(1)$ current. The operator versions of Maxwell equations take the form,

$$
g^{\alpha \beta} \nabla_{\alpha} \hat{F}_{\beta \mu}=q \hat{J}_{\mu}, \quad \nabla_{[\alpha} \hat{F}_{\mu \nu]}=0
$$

where the first one is an operator version of the first equation in (2.10), while the second one is a consequence of the anti-symmetry of the fields strength tensor, and where bracketed indices denote weighted anti-symmetrization. We shall combine these operator equations in a way that allows us to compute the vector field strength correlators conveniently. Taking the correlator of the first of these equations with itself results in an equation relating the correlators in (3.2), while taking the correlator of the second equation with the field strength tensor results in the homogeneous equation for the second correlator in (3.2),

$$
\begin{aligned}
& {\left[g^{\alpha \rho}(x) \nabla_{\alpha}\right] \times\left[g^{\beta \sigma}\left(x^{\prime}\right) \nabla_{\beta}^{\prime}\right] \times\left\langle\hat{F}_{\rho \mu}(x) \hat{F}_{\sigma \nu}\left(x^{\prime}\right)\right\rangle=q^{2}\left\langle\hat{J}_{\mu}(x) \hat{J}_{\nu}\left(x^{\prime}\right)\right\rangle,} \\
& \nabla_{[\alpha}\left\langle\hat{F}_{\mu \nu]}(x) \hat{F}_{\rho \sigma}\left(x^{\prime}\right)\right\rangle=0, \quad \nabla_{\beta]}^{\prime}\left\langle\hat{F}_{\mu \nu}(x) \hat{F}_{[\rho \sigma}\left(x^{\prime}\right)\right\rangle=0 .
\end{aligned}
$$

These equations are of a type similar to Dyson-Schwinger equations for Wightman functions. They are perfectly suited for our purposes of perturbative computation: the source on the right hand side of (3.5) - the $U(1)$ charge current correlator - comes multiplied by the expansion parameter $q^{2}$. Moreover, they allow for an immediate physical interpretation: the tree-level correlator of charge currents sources the oneloop correction to the field strength correlator. This is precisely the way in which we are going to perform the computation: we shall compute the tree-level current correlator induced on the scalar by the inflating space-time in Sec. 4, and then we shall use that result to correct the electric and magnetic field correlators in Sec. 5 .

\section{Current correlators}

The operator of the conserved $U(1)$ current is given by (2.11), where the fields are substituted by field operators,

$$
\hat{J}_{\mu}=i\left[\hat{\Phi}\left(\partial_{\mu} \hat{\Phi}^{\dagger}\right)-\hat{\Phi}^{\dagger}\left(\partial_{\mu} \hat{\Phi}\right)\right]+2 q \hat{A}_{\mu} \hat{\Phi}^{\dagger} \hat{\Phi}
$$

and it is covariantly conserved as an operator identity,

$$
0=g^{\mu \nu} \nabla_{\mu} \hat{J}_{\nu}
$$

Here we wish to compute the correlators of the components of the $U(1)$ current, $\left\langle\hat{J}_{\mu}(x) \hat{J}_{\nu}\left(x^{\prime}\right)\right\rangle$ with respect to the locally (and globally) neutral state, i.e. the state 
in which the expectation value of the current vanishes, $\left\langle\hat{J}_{\mu}(x)\right\rangle=0$. Even though neutral on average, that state still experiences quantum fluctuations, and hence the local charge also must fluctuate. This effect is captured by the components of the current correlators. We compute these to leading order in the coupling constant, which corresponds to neglecting its coupling to the vector. For the globally neutral state the leading order current correlators reduce to

$$
\left\langle\hat{J}_{\mu}(x) \hat{J}_{\nu}\left(x^{\prime}\right)\right\rangle=2 i \Delta\left(x ; x^{\prime}\right) \times \partial_{\mu} \partial_{\nu}^{\prime} i \Delta\left(x ; x^{\prime}\right)-2 \partial_{\mu} i \Delta\left(x ; x^{\prime}\right) \times \partial_{\nu}^{\prime} i \Delta\left(x ; x^{\prime}\right),
$$

where $i \Delta\left(x ; x^{\prime}\right)$ is the 2 -point function of the free complex scalar field, ${ }^{7}$

$$
i \Delta\left(x ; x^{\prime}\right)=\left\langle\hat{\Phi}^{\dagger}(x) \hat{\Phi}\left(x^{\prime}\right)\right\rangle .
$$

For a discussion on charge fluctuations in primordial inflation and their possible effects during and after inflation see [22, 23, 53, 54].

When the scalar two-point function (propagator) in (4.4) is known then it is a simple matter of acting on it with derivatives to obtain the charge current correlators in (4.3). However, the propagator for the minimally coupled massless scalar is not known for arbitrary FLRW space-times. ${ }^{8}$ One notable example is the power-law inflation space-time introduced in Sec. 2.1, for which scalar propagator has been reported in [55]. There the propagator for the Chernikov-Tagirov-Bunch-Davies (CTBD) state [56, 57], defined as the state that minimizes energy mode-per-mode in the asymptotic past, was computed in $D$ space-time dimensions. The well known IR divergence of the exact CTBD state in accelerating space-times [58, 59] (see also [60] for a recent review) is regulated by assuming that the IR modes below some scale $k_{0} \ll H_{0}$ have a suppressed spectrum compared to the CTBD one, and only the leading contribution in $k_{0}$ is kept. Here we need the $D=4$ limit of the propagator from [55] which reads,

$$
\begin{gathered}
i \Delta\left(x ; x^{\prime}\right)=\left[\frac{(1-\epsilon) H_{0}}{4 \pi}\right]^{2}\left\{\Gamma\left(\frac{3}{2}+\nu\right) \Gamma\left(\frac{3}{2}-\nu\right)\left(a a^{\prime}\right)^{-\epsilon} \times{ }_{2} F_{1}\left(\left\{\frac{3}{2}+\nu, \frac{3}{2}-\nu\right\},\{2\}, 1-\frac{y}{4}\right)\right. \\
\left.+\frac{\Gamma^{2}(2 \nu)}{(2 \nu-3) \Gamma^{2}\left(\frac{1}{2}+\nu\right)}\left[\frac{(1-\epsilon) H_{0}}{2 k_{0}}\right]^{2 \nu-3}\right\},
\end{gathered}
$$

where we have introduced the distance function,

$$
y\left(x ; x^{\prime}\right)=(1-\epsilon)^{2} \mathcal{H} \mathcal{H}^{\prime}\left[\|\Delta \vec{x}\|^{2}-\left(\eta-\eta^{\prime}\right)^{2}\right],
$$

\footnotetext{
${ }^{7}$ The 2-point function we consider has no time ordering in its definition, i.e. it is a Wightman rather than the Feynman 2-point function. However, as we are interested in correlators on superhorizon distances, using either suffices, and we do not distinguish between them explicitly.

${ }^{8}$ In the case of the conformally coupled scalar the propagator is known for all the FLRW spacetimes since it is not sensitive to the expansion, and takes the form of the rescaled flat space propagator.
} 
with $\Delta \vec{x}=\vec{x}-\vec{x}^{\prime}$, the parameters and their ranges are,

$$
\nu=\frac{(3-\epsilon)}{2(1-\epsilon)}, \quad \frac{3}{2} \leq \nu<\frac{5}{2} \quad \text { for } \quad 0 \leq \epsilon<\frac{1}{2} .
$$

and where $k_{0}$ is the effective IR regulator, ${ }^{9}$ satisfying $0<k_{0} \ll(1-\epsilon) H_{0}$. The dependence on the scale $k_{0}$ physically implies vast IR gravitational particle production, and $k_{0}$ should properly be seen as dependence on the initial conditions.

As we are interested in computing the correlators at superhorizon distances we will only need the asymptotic form of the propagator,

$$
\begin{aligned}
i \Delta\left(x ; x^{\prime}\right) & \stackrel{\overline{\mathrm{SH}}}{\sim}\left[\frac{(1-\epsilon) H_{0}}{4 \pi}\right]^{2} \frac{2^{3-2 \nu} \Gamma(2 \nu)}{\Gamma\left(\frac{1}{2}+\nu\right)} \times\left\{\frac{\Gamma(2 \nu)}{(2 \nu-3) \Gamma\left(\frac{1}{2}+\nu\right)}\left[\frac{(1-\epsilon) H_{0}}{k_{0}}\right]^{2 \nu-3}\right. \\
& +\left[(1-\epsilon) H_{0}\|\Delta \vec{x}\|\right]^{2 \nu-3}\left[\Gamma\left(\frac{3}{2}-\nu\right)+\Gamma\left(\frac{5}{2}-\nu\right)\left(\frac{\mathcal{H}}{\mathcal{H}^{\prime}}+\frac{\mathcal{H}^{\prime}}{\mathcal{H}}\right) \frac{1}{(1-\epsilon)^{2} \mathcal{H} \mathcal{H}^{\prime}\|\Delta \vec{x}\|^{2}}\right. \\
& \left.\left.+\Gamma\left(\frac{7}{2}-\nu\right)\left(\frac{1}{2}\left(\frac{\mathcal{H}}{\mathcal{H}^{\prime}}+\frac{\mathcal{H}^{\prime}}{\mathcal{H}}\right)^{2}-\frac{1}{\nu-1}\right) \frac{1}{(1-\epsilon)^{4}\left(\mathcal{H} \mathcal{H}^{\prime}\right)^{2}\|\Delta \vec{x}\|^{4}}\right]\right\}
\end{aligned}
$$

Plugging this expansion into (4.3) yields the desired leading order contributions to superhorizon current correlators,

$$
\begin{aligned}
& \left\langle\hat{J}_{0}(x) \hat{J}_{0}\left(x^{\prime}\right)\right\rangle \stackrel{\overline{\mathrm{SH}}}{\sim} \mathcal{N}\left[\frac{(1-\epsilon) H_{0}}{4 \pi}\right]^{4} \times \frac{\left[(1-\epsilon) H_{0}\|\Delta \vec{x}\|\right]^{4 \nu-6}}{\|\Delta \vec{x}\|^{2}} \times \frac{1}{(1-\epsilon)^{2} \mathcal{H} \mathcal{H}^{\prime}\|\Delta \vec{x}\|^{2}} \\
& \times \frac{(\nu-2)}{(\nu-1)}\left[\frac{1}{\left(k_{0}\|\Delta \vec{x}\|\right)^{2 \nu-3}}+\frac{2(4 \nu-7) \Gamma\left(\frac{5}{2}-\nu\right) \Gamma\left(\frac{1}{2}+\nu\right)}{(2 \nu-5)(\nu-2) \Gamma(2 \nu)}\right], \\
& \left\langle\hat{J}_{0}(x) \hat{J}_{i}\left(x^{\prime}\right)\right\rangle \stackrel{\overline{\mathrm{SH}}}{\sim}-\mathcal{N}\left[\frac{(1-\epsilon) H_{0}}{4 \pi}\right]^{4} \times \frac{\left[(1-\epsilon) H_{0}\|\Delta \vec{x}\|\right]^{4 \nu-6}}{\|\Delta \vec{x}\|^{2}} \times \frac{1}{(1-\epsilon) \mathcal{H}\|\Delta \vec{x}\|} \\
& \times\left[\frac{1}{\left(k_{0}\|\Delta \vec{x}\|\right)^{2 \nu-3}}+\frac{4 \Gamma\left(\frac{5}{2}-\nu\right) \Gamma\left(\frac{1}{2}+\nu\right)}{(2 \nu-5) \Gamma(2 \nu)}\right] \times \frac{\Delta x_{i}}{\|\Delta \vec{x}\|}, \\
& \left\langle\hat{J}_{i}(x) \hat{J}_{j}\left(x^{\prime}\right)\right\rangle \stackrel{\overline{\mathrm{SH}}}{\sim}-\mathcal{N}\left[\frac{(1-\epsilon) H_{0}}{4 \pi}\right]^{4} \times \frac{\left[(1-\epsilon) H_{0}\|\Delta \vec{x}\|\right]^{4 \nu-6}}{\|\Delta \vec{x}\|^{2}} \times \\
& \times\left\{\frac{1}{(2 \nu-5)}\left[\frac{1}{\left(k_{0}\|\Delta \vec{x}\|\right)^{2 \nu-3}}-\frac{2 \Gamma\left(\frac{5}{2}-\nu\right) \Gamma\left(\frac{1}{2}+\nu\right)}{\Gamma(2 \nu)}\right] \delta_{i j}\right. \\
& \left.+\left[\frac{1}{\left(k_{0}\|\Delta \vec{x}\|\right)^{2 \nu-3}}+\frac{4 \Gamma\left(\frac{5}{2}-\nu\right) \Gamma\left(\frac{1}{2}+\nu\right)}{(2 \nu-5) \Gamma(2 \nu)}\right] \frac{\Delta x_{i} \Delta x_{j}}{\|\Delta \vec{x}\|^{2}}\right\},
\end{aligned}
$$

\footnotetext{
${ }^{9}$ The scalar propagator in (4.5) is valid for $k_{0}\|\Delta \vec{x}\| \ll 1$. Beyond this region its form changes and it typically decays exponentially with spatial distance, depending on the specifics of the deep IR sector of the state.
} 
where the overall normalization constant is

$$
\mathcal{N}=\frac{8 \Gamma(2 \nu) \Gamma\left(\frac{7}{2}-\nu\right)}{(2 \nu-3) \Gamma\left(\frac{1}{2}+\nu\right)}\left[\frac{2^{3-2 \nu} \Gamma(2 \nu)}{\Gamma\left(\frac{1}{2}+\nu\right)}\right]^{2} .
$$

The conservation of the current operator (4.2) implies consistency conditions on both legs of the current correlators,

$$
\begin{aligned}
& 0=-\partial_{0}\left\langle\hat{J}_{0}(x) \hat{J}_{\mu}\left(x^{\prime}\right)\right\rangle-2 \mathcal{H}\left\langle\hat{J}_{0}(x) \hat{J}_{\mu}\left(x^{\prime}\right)\right\rangle+\partial_{i}\left\langle\hat{J}_{i}(x) \hat{J}_{\mu}\left(x^{\prime}\right)\right\rangle, \\
& 0=-\partial_{0}^{\prime}\left\langle\hat{J}_{\mu}(x) \hat{J}_{0}\left(x^{\prime}\right)\right\rangle-2 \mathcal{H}^{\prime}\left\langle\hat{J}_{\mu}(x) \hat{J}_{0}\left(x^{\prime}\right)\right\rangle+\partial_{i}^{\prime}\left\langle\hat{J}_{\mu}(x) \hat{J}_{i}\left(x^{\prime}\right)\right\rangle,
\end{aligned}
$$

which are exactly satisfied by (4.9)-(4.11). Note that, apart from the numerical coefficients and the tensor structures, at late times, and large spatial separations, the $(00)$ correlator is down by a factor $\left(\mathcal{H}^{\prime}\|\Delta \vec{x}\|\right)$ compared to the $(0 i)$ correlator, which is down by a factor $(\mathcal{H}\|\Delta \vec{x}\|)$ compared to the $(i j)$ correlator. This hierarchy is a generic feature expounded on in Sec. 6.

In the language of the Schwinger-Keldysh formalism of QFT, the charge correlators we have computed correspond to the $(-+)$ component of the one-loop vacuum polarization,

$$
i\left[{ }_{\mu}^{-} \Pi_{\nu}^{+}\right]\left(x ; x^{\prime}\right)=q^{2}\left(a a^{\prime}\right)^{2}\left\langle\hat{J}_{\mu}(x) \hat{J}_{\nu}\left(x^{\prime}\right)\right\rangle,
$$

which at superhorizon distances also corresponds to the $(++)$ component,

$$
i\left[{ }_{\mu}^{+} \Pi_{\nu}^{+}\right]\left(x ; x^{\prime}\right)=q^{2}\left(a a^{\prime}\right)^{2}\left\langle\mathcal{T}\left[\hat{J}_{\mu}(x) \hat{J}_{\nu}\left(x^{\prime}\right)\right]\right\rangle \stackrel{\overline{S H}}{\sim} q^{2}\left(a a^{\prime}\right)^{2}\left\langle\hat{J}_{\mu}(x) \hat{J}_{\nu}\left(x^{\prime}\right)\right\rangle,
$$

where $\mathcal{T}$ denotes time-ordering. The factors of the scale factor are due to the definition of vacuum polarization. The diagrams corresponding to the one-loop vacuum polarization are given in Fig. 4. It is only the non-local diagram that contributes
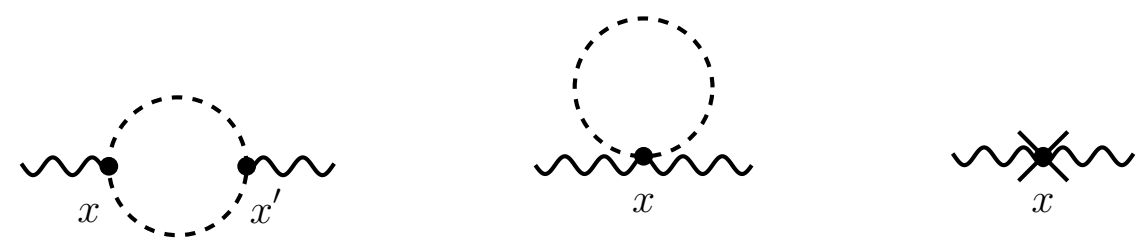

Figure 4. Diagrams contributing to full one-loop vacuum polarization: left: bubble diagram, middle: seagull diagram, right: photon field strength renormalization counterterm. Dashed lines represent the scalar propagator, and wavy lines the amputated vector propagator. The endpoints are assumed to carry a space-time label, a vector index, and a Schwinger-Keldysh polarity label.

to the $(-+)$ component, while all three contribute to the $(++)$ component. However, the two local diagrams contribute only in coincidence, $\propto \delta^{4}\left(x-x^{\prime}\right)$, and not for superhorizon separations. We neglect their contributions on two accounts: (i) it 
was shown in $[27,31]$ that in de Sitter space the only local contribution contributing to the vacuum polarization is the conformal anomaly, whose effect is weak [14], and (ii) we expect them to contribute only subdominantly as they correspond to homogeneous solutions of Eqs. (5.12)-(5.12) discussed in the following section.

Determining whether correlators (4.9)-(4.11) represent a big or a small effect is not as straightforward since (i) there is no lower-order effect to compare to, and (ii) the redshifting of the correlators depends on whether we present them with raised or lowered indices. A more meaningful comparison can be done on the level of electric and magnetic field correlators given in Sec. 5, where one can compare them to treelevel contribution (3.3).

The exact de Sitter limit, $\epsilon \rightarrow 0$, of the current correlators (4.11)-(4.9) takes a simpler form,

$$
\begin{gathered}
\left\langle\hat{J}_{0}(x) \hat{J}_{0}\left(x^{\prime}\right)\right\rangle \stackrel{\overline{\mathrm{SH}}}{\sim} \frac{H_{0}^{4}}{8 \pi^{4}\|\Delta \vec{x}\|^{2}} \times \frac{1}{\mathcal{H} \mathcal{H}^{\prime}\|\Delta \vec{x}\|^{2}} \times\left[-3+2 \gamma_{\mathrm{E}}+2 \ln \left(k_{0}\|\Delta \vec{x}\|\right)\right] \\
\left\langle\hat{J}_{0}(x) \hat{J}_{i}\left(x^{\prime}\right)\right\rangle \stackrel{\overline{\mathrm{SH}}}{\sim} \frac{H_{0}^{4}}{8 \pi^{4}\|\Delta \vec{x}\|^{2}} \times \frac{1}{\mathcal{H}\|\Delta \vec{x}\|} \times\left[-2 \ln \left(k_{0}\|\Delta \vec{x}\|\right)-2 \gamma_{\mathrm{E}}+1\right] \frac{\Delta x_{i}}{\|\Delta \vec{x}\|} \\
\left\langle\hat{J}_{i}(x) \hat{J}_{j}\left(x^{\prime}\right)\right\rangle \stackrel{\mathrm{SH}}{\sim} \frac{H_{0}^{4}}{8 \pi^{4}\|\Delta \vec{x}\|^{2}}\left\{\left[-\ln \left(k_{0}\|\Delta \vec{x}\|\right)-\gamma_{\mathrm{E}}+1\right] \delta_{i j}\right. \\
\left.+\left[2 \ln \left(k_{0}\|\Delta \vec{x}\|\right)+2 \gamma_{\mathrm{E}}-1\right] \frac{\Delta x_{i} \Delta x_{j}}{\|\Delta \vec{x}\|^{2}}\right\}
\end{gathered}
$$

where $\gamma_{\mathrm{E}}$ is the Euler-Mascheroni constant. It provides an important consistency check with the previously reported results, as it reproduces the superhorizon limit of the vacuum polarization from [27] (Eq. (73) from their paper), provided that we make the identification $k_{0}=H_{0} e^{1-\gamma_{\mathrm{E}}}$, and taking the factors from (4.15) into account.

\section{$5 \quad$ Field strength correlators}

Charge current correlators are not the best object to characterize the physical behaviour. At tree-level they are zero so we have nothing to compare with when asking how big they are. More appropriate quantities are correlators of vector field strength tensor,

$$
\left\langle\hat{F}_{\mu \nu}(x) \hat{F}_{\rho \sigma}\left(x^{\prime}\right)\right\rangle
$$

where $\hat{F}_{\mu \nu}=\partial_{\mu} \hat{A}_{\nu}-\partial_{\nu} \hat{A}_{\mu}$. It is better to present this correlator in the form of electric and magnetic field correlators, where the E\&M field operators are, respectively,

$$
\hat{E}_{i}=\hat{F}_{0 i}, \quad \hat{B}_{i}=-\frac{1}{2} \varepsilon_{i j k} \hat{F}_{j k},
$$


and where $\varepsilon_{i j k}$ is the 3 -dimensional Levi-Civita symbol. The E\&M correlators exist even in the free theory because of the quantum fluctuations. Due to the conformal coupling, the free vector field does not sense the expansion of the FLRW space-time, and the electric and magnetic field correlators take the same form (3.3) as in flat space,

$$
\begin{aligned}
& \left\langle\hat{E}_{i}(\eta, \vec{x}) \hat{E}_{j}\left(\eta, \vec{x}^{\prime}\right)\right\rangle=\frac{1}{\pi^{2}\|\Delta \vec{x}\|^{4}}\left[-\delta_{i j}+2 \frac{\Delta x_{i} \Delta x_{j}}{\|\Delta \vec{x}\|^{2}}\right], \\
& \left\langle\hat{E}_{i}(\eta, \vec{x}) \hat{B}_{j}\left(\eta, \vec{x}^{\prime}\right)\right\rangle=0 \\
& \left\langle\hat{B}_{i}(\eta, \vec{x}) \hat{B}_{j}\left(\eta, \vec{x}^{\prime}\right)\right\rangle=\frac{1}{\pi^{2}\|\Delta \vec{x}\|^{4}}\left[-\delta_{i j}+2 \frac{\Delta x_{i} \Delta x_{j}}{\|\Delta \vec{x}\|^{2}}\right] .
\end{aligned}
$$

As a consequence of coupling to the charged scalar this does not remain true - the scalar mediates the effects of the expansion to the vector field.

We quantify the influence of the charged scalar on the vector by computing the one-loop correction to E\&M field correlators, which is the first correction in the coupling constant $q^{2}$. This we do by solving perturbatively a variant of the equations (3.5) and (3.6) for the correlators derived in Sec. 3. Instead of the covariant version, it is better to write them in terms of E\&M fields, for which the Maxwell's equations read,

$$
\begin{array}{ll}
\partial_{i} \hat{E}_{i}=-a^{2} q \hat{J}_{0}, & \partial_{0} \hat{E}_{i}-\varepsilon_{i j k} \partial_{j} \hat{B}_{k}=-a^{2} q \hat{J}_{i} . \\
\partial_{i} \hat{B}_{i}=0, & \partial_{0} \hat{B}_{i}+\varepsilon_{i j k} \partial_{j} \hat{E}_{k}=0 .
\end{array}
$$

The first line corresponds to the inhomogeneous equation in (3.4) containing respectively Gauss' law and Ampère's law, while the second line corresponds to the homogeneous equations in (3.4) containing respectively the law of vanishing magnetic charge, and Faraday's law. Instead of forming the correlators of these first order equations directly, it is more convenient to use the second-order sourced wave equations,

$$
\begin{aligned}
& \left(\partial_{0}^{2}-\nabla^{2}\right) \hat{E}_{i}=-\partial_{0}\left(a^{2} q \hat{J}_{i}\right)+a^{2} \partial_{i} q \hat{J}_{0}, \\
& \left(\partial_{0}^{2}-\nabla^{2}\right) \hat{B}_{i}=\varepsilon_{i j k} \partial_{j} a^{2} q \hat{J}_{k}
\end{aligned}
$$

in which E\&M fields decouple. Forming the correlators between different combinations of the two equations above results in,

$$
\begin{aligned}
&\left(\partial_{0}^{2}-\nabla^{2}\right)\left(\partial_{0}^{\prime 2}-\nabla^{\prime 2}\right)\left\langle\hat{E}_{i}(x) \hat{E}_{j}\left(x^{\prime}\right)\right\rangle \\
&=q^{2} \partial_{0} \partial_{0}^{\prime}\left[\left(a a^{\prime}\right)^{2}\left\langle\hat{J}_{i}(x) \hat{J}_{j}\left(x^{\prime}\right)\right\rangle\right]-q^{2} \partial_{0} \partial_{j}^{\prime}\left[\left(a a^{\prime}\right)^{2}\left\langle\hat{J}_{i}(x) \hat{J}_{0}\left(x^{\prime}\right)\right\rangle\right] \\
& \quad-q^{2} \partial_{i} \partial_{0}^{\prime}\left[\left(a a^{\prime}\right)^{2}\left\langle\hat{J}_{0}(x) \hat{J}_{j}\left(x^{\prime}\right)\right\rangle\right]+q^{2} \partial_{i} \partial_{j}^{\prime}\left[\left(a a^{\prime}\right)^{2}\left\langle\hat{J}_{0}(x) \hat{J}_{0}\left(x^{\prime}\right)\right\rangle\right],
\end{aligned}
$$




$$
\begin{aligned}
\left(\partial_{0}^{2}-\nabla^{2}\right) & \left(\partial_{0}^{\prime 2}-\nabla^{\prime 2}\right)\left\langle\hat{E}_{i}(x) \hat{B}_{j}\left(x^{\prime}\right)\right\rangle \\
& =-q^{2} \varepsilon_{j k l} \partial_{0} \partial_{k}^{\prime}\left[\left(a a^{\prime}\right)^{2}\left\langle\hat{J}_{i}(x) \hat{J}_{l}\left(x^{\prime}\right)\right\rangle\right]+q^{2} \varepsilon_{j k l} \partial_{i} \partial_{k}^{\prime}\left[\left(a a^{\prime}\right)^{2}\left\langle\hat{J}_{0}(x) \hat{J}_{l}\left(x^{\prime}\right)\right\rangle\right] \\
\left(\partial_{0}^{2}-\nabla^{2}\right) & \left(\partial_{0}^{\prime 2}-\nabla^{\prime 2}\right)\left\langle\hat{B}_{i}(x) \hat{B}_{j}\left(x^{\prime}\right)\right\rangle \\
& =q^{2} \varepsilon_{i k l} \varepsilon_{j m n} \partial_{k} \partial_{m}^{\prime}\left[\left(a a^{\prime}\right)^{2}\left\langle\hat{J}_{l}(x) \hat{J}_{n}\left(x^{\prime}\right)\right\rangle\right]
\end{aligned}
$$

The utility of these particular equations becomes apparent when we apply them to solving for superhorizon correlators. Firstly, note that in the superhorizon limit a spatial derivative introduces another factor of $1 /\|\Delta \vec{x}\|$, while a time derivative introduces another factor of $\mathcal{H}$, and therefore the spatial derivatives on the right hand side of equations above can be dropped in favour of time derivatives. Secondly, all current correlators on the right hand side, except for the $(i j)$ component, may be dropped due to the superhorizon hierarchy between them discussed in Sec. 4,

$$
\begin{aligned}
& \left(\partial_{0} \partial_{0}^{\prime}\right)^{2}\left\langle\hat{E}_{i}(x) \hat{E}_{j}\left(x^{\prime}\right)\right\rangle \stackrel{\overline{\mathrm{SH}}}{\sim} q^{2} \partial_{0} \partial_{0}^{\prime}\left[\left(a a^{\prime}\right)^{2}\left\langle\hat{J}_{i}(x) \hat{J}_{j}\left(x^{\prime}\right)\right\rangle\right] \\
& \left(\partial_{0} \partial_{0}^{\prime}\right)^{2}\left\langle\hat{E}_{i}(x) \hat{B}_{j}\left(x^{\prime}\right)\right\rangle \stackrel{\overline{\mathrm{SH}}}{\sim}-q^{2} \varepsilon_{j k l} \partial_{0} \partial_{k}^{\prime}\left[\left(a a^{\prime}\right)^{2}\left\langle\hat{J}_{i}(x) \hat{J}_{l}\left(x^{\prime}\right)\right\rangle\right] \\
& \left(\partial_{0} \partial_{0}^{\prime}\right)^{2}\left\langle\hat{B}_{i}(x) \hat{B}_{j}\left(x^{\prime}\right)\right\rangle \stackrel{\overline{\mathrm{SH}}}{\sim} q^{2} \varepsilon_{i k l} \varepsilon_{j m n} \partial_{k} \partial_{m}^{\prime}\left[\left(a a^{\prime}\right)^{2}\left\langle\hat{J}_{l}(x) \hat{J}_{n}\left(x^{\prime}\right)\right\rangle\right] .
\end{aligned}
$$

Furthermore, the $(\mathrm{ij})$ component (4.11) of the current correlator is time-independent, making the task of inverting the time derivatives on the left-hand side straightforward. Making use of (2.5),

$$
\begin{aligned}
& \left\langle\hat{E}_{i}(x) \hat{E}_{j}\left(x^{\prime}\right)\right\rangle \stackrel{\overline{\mathrm{SH}}}{\sim} q^{2} \frac{\left(a a^{\prime}\right)^{1+\epsilon}}{(1+\epsilon)^{2} H_{0}^{2}}\left\langle\hat{J}_{i}(x) \hat{J}_{j}\left(x^{\prime}\right)\right\rangle, \\
& \left\langle\hat{E}_{i}(x) \hat{B}_{j}\left(x^{\prime}\right)\right\rangle \stackrel{\overline{\mathrm{SH}}}{\sim}-q^{2} \frac{a^{1+\epsilon}\left(a^{\prime}\right)^{2 \epsilon}}{2 \epsilon(1+\epsilon)^{2} H_{0}^{3}} \varepsilon_{j k l} \partial_{k}^{\prime}\left\langle\hat{J}_{i}(x) \hat{J}_{l}\left(x^{\prime}\right)\right\rangle, \\
& \left\langle\hat{B}_{i}(x) \hat{B}_{j}\left(x^{\prime}\right)\right\rangle \stackrel{\overline{\mathrm{SH}}}{\sim} q^{2} \frac{\left(a a^{\prime}\right)^{2 \epsilon}}{4 \epsilon^{2}(1+\epsilon)^{2} H_{0}^{4}} \varepsilon_{i k l} \varepsilon_{j m n} \partial_{k} \partial_{m}^{\prime}\left\langle\hat{J}_{l}(x) \hat{J}_{n}\left(x^{\prime}\right)\right\rangle .
\end{aligned}
$$

Note that these expressions are valid for late times in power-law inflation, when $\ln (a)>$ $1 / \epsilon$. The de Sitter limit $\epsilon \rightarrow 0$ cannot be taken directly in these late-time results on the account of factors of $\epsilon$ in the denominator. This tells us that the subleading term in the expansion, which is negligible at late times in power-law inflation, becomes equally relevant in this limit. However, instead of having to work out the subleading terms, it is simpler to invert equations (5.13)-(5.15) in the de Sitter limit directly, which we do at the end of this section.

The solutions (5.16)-(5.18) pass the consistency checks as they satisfy the various relations descending from the linear Maxwell equations (5.6) and (5.7). In particular 
relations descending from the inhomogeneous equations,

$$
\begin{aligned}
q^{2}\left(a a^{\prime}\right)^{2}\left\langle\hat{J}_{0}(x) \hat{J}_{0}\left(x^{\prime}\right)\right\rangle \stackrel{\overline{\mathrm{SH}}}{\sim} \partial_{i} \partial_{j}^{\prime}\left\langle\hat{E}_{i}(x) \hat{E}_{j}\left(x^{\prime}\right)\right\rangle \\
q^{2}\left(a a^{\prime}\right)^{2}\left\langle\hat{J}_{0}(x) \hat{J}_{i}\left(x^{\prime}\right)\right\rangle \stackrel{\overline{\mathrm{SH}}}{\sim} \partial_{j} \partial_{0}^{\prime}\left\langle\hat{E}_{j}(x) \hat{E}_{i}\left(x^{\prime}\right)\right\rangle+\partial_{j} \epsilon_{i k l} \partial_{k}^{\prime}\left\langle\hat{E}_{j}(x) \hat{B}_{l}\left(x^{\prime}\right)\right\rangle \\
q^{2}\left(a a^{\prime}\right)^{2}\left\langle\hat{J}_{i}(x) \hat{J}_{j}\left(x^{\prime}\right)\right\rangle \stackrel{\overline{\mathrm{SH}}}{\sim} \partial_{0} \partial_{0}^{\prime}\left\langle\hat{E}_{i}(x) \hat{E}_{j}\left(x^{\prime}\right)\right\rangle+\epsilon_{j m n} \partial_{0} \partial_{m}^{\prime}\left\langle\hat{E}_{i}(x) \hat{B}_{n}\left(x^{\prime}\right)\right\rangle \\
\\
+\epsilon_{i k l} \partial_{k} \partial_{0}^{\prime}\left\langle\hat{B}_{l}(x) \hat{E}_{j}\left(x^{\prime}\right)\right\rangle+\epsilon_{i k l} \epsilon_{j m n} \partial_{k} \partial_{m}^{\prime}\left\langle\hat{B}_{l}(x) \hat{B}_{n}\left(x^{\prime}\right)\right\rangle
\end{aligned}
$$

are satisfied to leading order, and the relations descending from homogeneous equations,

$$
\begin{aligned}
& 0=\varepsilon_{j k l} \partial_{k}^{\prime}\left\langle\hat{E}_{i}(x) \hat{E}_{l}\left(x^{\prime}\right)\right\rangle+\partial_{0}^{\prime}\left\langle\hat{E}_{i}(x) \hat{B}_{j}\left(x^{\prime}\right)\right\rangle, \\
& 0=\varepsilon_{i k l} \partial_{k}\left\langle\hat{E}_{l}(x) \hat{B}_{j}\left(x^{\prime}\right)\right\rangle+\partial_{0}\left\langle\hat{B}_{i}(x) \hat{B}_{j}\left(x^{\prime}\right)\right\rangle, \\
& 0=\partial_{j}^{\prime}\left\langle\hat{E}_{i}(x) \hat{B}_{j}\left(x^{\prime}\right)\right\rangle \\
& 0=\partial_{i}\left\langle\hat{B}_{i}(x) \hat{B}_{j}\left(x^{\prime}\right)\right\rangle
\end{aligned}
$$

are satisfied exactly. Additional relations can be generated by exploiting symmetries of the correlators under the excange of indices and ordering of the fields.

The computation presented here is equivalent to the standard QFT computation, where we would first compute the one-loop correction to the Wightman two-point function of the vector field $\left\langle\hat{A}_{\mu}(x) \hat{A}_{\nu}\left(x^{\prime}\right)\right\rangle$, given by the diagrams in Fig. 5 , and then act with derivative operators to obtain the E\&M correlators,

$$
\left\langle\hat{F}_{\mu \nu}(x) \hat{F}_{\rho \sigma}\left(x^{\prime}\right)\right\rangle=4\left(\partial_{[\mu} \delta_{\nu]}^{\alpha}\right)\left(\partial_{[\rho}^{\prime} \delta_{\sigma]}^{\beta}\right)\left\langle\hat{A}_{\alpha}(x) \hat{A}_{\beta}\left(x^{\prime}\right)\right\rangle .
$$

As we are only interested in superhorizon behaviour, this way we have avoided having to go through a more complicated computation of first computing a gauge-dependent object and only then acting with derivative operators to project out the physical information. In fact, it is not possible to perform the dimensionally regulated oneloop computation in power-law inflation, since the photon propagators have not been reported in $D$-dimensional power-law inflation, and would have to be computed first. Our computation captures the effects of the first diagram in Fig. 5, which is the important one at superhorizon separations, and represents the reaction of the photon to the charge current fluctuations of the complex scalar induced by the expansion of the space-time (see Fig. 2). The explicit expressions for the one-loop corrected equal-time correlators of electric and magnetic fields at super-Hubble separations 

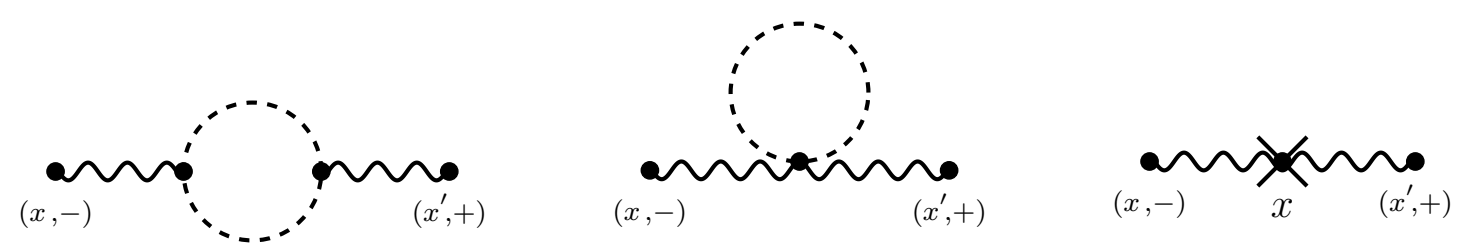

Figure 5. Diagrams contributing to one-loop corrections of the vector field two-point function $\left\langle\hat{A}_{\mu}(x) \hat{A}_{\nu}\left(x^{\prime}\right)\right\rangle$; left: bubble diagram, middle: seagull diagram, right: photon field strength renormalization counterterm. Dashed lines represent the tree-level scalar propagator, and wavy lines the photon propagator. The Schwinger-Keldysh polarities of the endpoints denote these are the diagams corresponding to the Wightman two-point function.

follow from (5.16)-(5.18),

$$
\begin{aligned}
& \left\langle\hat{E}_{i}(\eta, \vec{x}) \hat{E}_{j}\left(\eta, \vec{x}^{\prime}\right)\right\rangle \stackrel{\mathrm{SH}}{\sim} \\
& \frac{1}{\pi^{2}\|\Delta \vec{x}\|^{4}}\left\{\delta_{i j}\left[-1-q^{2}[(1-\epsilon) \mathcal{H}\|\Delta \vec{x}\|]^{4 \nu-4} \mathcal{K}_{1}\left(k_{0}\|\Delta \vec{x}\|\right)\right],\right. \\
& \left.+\frac{\Delta x_{i} \Delta x_{j}}{\|\Delta \vec{x}\|^{2}}\left[2-q^{2}[(1-\epsilon) \mathcal{H}\|\Delta \vec{x}\|]^{4 \nu-4} \mathcal{K}_{2}\left(k_{0}\|\Delta \vec{x}\|\right)\right]\right\} \\
& \left\langle\hat{E}_{i}(\eta, \vec{x}) \hat{B}_{j}\left(\eta, \vec{x}^{\prime}\right)\right\rangle \stackrel{\mathrm{SH}}{\sim} \\
& \frac{1}{\pi^{2}\|\Delta \vec{x}\|^{4}}\left\{\varepsilon_{i j k} \frac{\Delta x_{k}}{\|\Delta \vec{x}\|}\left[0-\frac{q^{2}}{2 \epsilon}[(1-\epsilon) \mathcal{H}\|\Delta \vec{x}\|]^{4 \nu-5}\right] \mathcal{K}_{3}\left(k_{0}\|\Delta \vec{x}\|\right)\right\}, \\
& \left\langle\hat{B}_{i}(\eta, \vec{x}) \hat{B}_{j}\left(\eta, \vec{x}^{\prime}\right)\right\rangle \stackrel{\mathrm{SH}}{\sim} \\
& \frac{1}{\pi^{2}\|\Delta \vec{x}\|^{4}}\left\{\delta_{i j}\left[-1+\frac{q^{2}}{4 \epsilon^{2}}[(1-\epsilon) \mathcal{H}\|\Delta \vec{x}\|]^{4 \nu-6} \mathcal{K}_{4}\left(k_{0}\|\Delta \vec{x}\|\right)\right]\right. \\
& \left.+\frac{\Delta x_{i} \Delta x_{j}}{\|\Delta \vec{x}\|^{2}}\left[2+\frac{q^{2}}{4 \epsilon^{2}}[(1-\epsilon) \mathcal{H}\|\Delta \vec{x}\|]^{4 \nu-6} \mathcal{K}_{5}\left(k_{0}\|\Delta \vec{x}\|\right)\right]\right\}
\end{aligned}
$$

where the short hand notation is,

$$
\begin{aligned}
& \mathcal{K}_{1}\left(k_{0}\|\Delta \vec{x}\|\right)=\frac{\mathcal{N}}{2^{8} \pi^{2}}\left(\frac{1-\epsilon}{1+\epsilon}\right)^{2} \frac{1}{(2 \nu-5)}\left[\frac{1}{\left(k_{0}\|\Delta \vec{x}\|\right)^{2 \nu-3}}-\frac{2 \Gamma\left(\frac{5}{2}-\nu\right) \Gamma\left(\frac{1}{2}+\nu\right)}{\Gamma(2 \nu)}\right], \\
& \mathcal{K}_{2}\left(k_{0}\|\Delta \vec{x}\|\right)=\frac{\mathcal{N}}{2^{8} \pi^{2}}\left(\frac{1-\epsilon}{1+\epsilon}\right)^{2}\left[\frac{1}{\left(k_{0}\|\Delta \vec{x}\|\right)^{2 \nu-3}}+\frac{4 \Gamma\left(\frac{5}{2}-\nu\right) \Gamma\left(\frac{1}{2}+\nu\right)}{(2 \nu-5) \Gamma(2 \nu)}\right] \\
& \mathcal{K}_{3}\left(k_{0}\|\Delta \vec{x}\|\right)=\frac{(1-\epsilon) \mathcal{N}}{2^{8} \pi^{2}}\left(\frac{1-\epsilon}{1+\epsilon}\right)^{2}\left[(4 \nu-8) \mathcal{K}_{1}+k_{0}\|\Delta \vec{x}\| \mathcal{K}_{1}^{\prime}-\mathcal{K}_{2}\right]
\end{aligned}
$$




$$
\begin{aligned}
& \mathcal{K}_{4}\left(k_{0}\|\Delta \vec{x}\|\right)=\frac{(1-\epsilon) \mathcal{N}}{2^{8} \pi^{2}}\left(\frac{1-\epsilon}{1+\epsilon}\right)^{2}\left[(4 \nu-8) \mathcal{K}_{3}+k_{0}\|\Delta \vec{x}\| \mathcal{K}_{3}^{\prime}\right], \\
& \mathcal{K}_{5}\left(k_{0}\|\Delta \vec{x}\|\right)=\frac{(1-\epsilon) \mathcal{N}}{2^{8} \pi^{2}}\left(\frac{1-\epsilon}{1+\epsilon}\right)^{2}\left[-(4 \nu-10) \mathcal{K}_{3}-k_{0}\|\Delta \vec{x}\| \mathcal{K}_{3}^{\prime}\right],
\end{aligned}
$$

and where primes on $\mathcal{K}$ 's here denote a derivative with respect to the argument. Importantly, note that no matter how small the coupling constant $q$ is, given enough time the one-loop correction will dominate over the tree-level contribution due to power-law secular growth. Moreover, note that one-loop corrections satisfy a hierarchy. Apart from the numerical coefficients, and the tensor structures, the $(B B)$ correlator is down by a factor $(\mathcal{H}\|\Delta \vec{x}\|)$ compared to the $(E B)$ correlator, which is down by a factor $(\mathcal{H}\|\Delta \vec{x}\|)$ compared to the $(E E)$ correlator. We will see in Sec. 6 this is not a peculiarity of the model, but rather a generic feature of E\&M correlators in inflation.

We conclude this section by working out the correlators in the de Sitter limit $\epsilon=$ 0 . Note that in power-law inflation, apart from the overall factor $1 /\|\Delta \vec{x}\|^{4}$ and the IR sensitivity $k_{0}\|\Delta \vec{x}\|$, the corrections (5.27)-(5.29) depend on coordinates only as $(\mathcal{H}\|\Delta \vec{x}\|)$. This must be the case in de Sitter space as well, where this quantity is proportional to the physical distance. Assuming this dependence and going through the exercise of inverting Eqs. (5.13)-(5.15) for $\epsilon=0$ produces,

$$
\begin{aligned}
\left\langle\hat{E}_{i}(\eta, \vec{x}) \hat{E}_{j}\left(\eta, \vec{x}^{\prime}\right)\right\rangle \stackrel{\mathrm{SH}}{\sim} \frac{1}{\pi^{2}\|\Delta \vec{x}\|^{4}}\left\{1+\frac{q^{2}}{8 \pi^{2}}(\mathcal{H}\|\Delta \vec{x}\|)^{2}\left[\left(-\ln \left(k_{0}\|\Delta \vec{x}\|\right)-\gamma_{\mathrm{E}}+1\right) \delta_{i j}\right.\right. \\
\left.\left.+\left(2 \ln \left(k_{0}\|\Delta \vec{x}\|\right)+2 \gamma_{\mathrm{E}}-1\right) \frac{\Delta x_{i} \Delta x_{j}}{\|\Delta \vec{x}\|^{2}}\right]\right\}, \\
\left\langle\hat{E}_{i}(\eta, \vec{x}) \hat{B}_{j}\left(\eta, \vec{x}^{\prime}\right)\right\rangle \stackrel{\mathrm{SH}}{\sim} \frac{1}{\pi^{2}\|\Delta \vec{x}\|^{4}}\left\{0-\frac{q^{2}}{4 \pi^{2}}(\mathcal{H}\|\Delta \vec{x}\|) \ln (\mathcal{H}\|\Delta \vec{x}\|) \times \varepsilon_{i j k} \frac{\Delta x_{k}}{\|\Delta \vec{x}\|}\right\}, \\
\left\langle\hat{B}_{i}(\eta, \vec{x}) \hat{B}_{j}\left(\eta, \vec{x}^{\prime}\right)\right\rangle \stackrel{\mathrm{SH}}{\sim} \frac{1}{\pi^{2}\|\Delta \vec{x}\|^{4}}\left\{1+\frac{q^{2}}{2 \pi^{2}} \ln ^{2}(\mathcal{H}\|\Delta \vec{x}\|)\left[\delta_{i j}-2 \frac{\Delta x_{i} \Delta x_{i}}{\|\Delta \vec{x}\|^{2}}\right]\right\} .
\end{aligned}
$$

Note that the hierarchy here is supplemented by additional factors of $\ln (\mathcal{H}\|\Delta \vec{x}\|)$. The above results in the de Sitter limit can be checked independently by performing the standard one-loop computation for the vector field two-point function in the Schwinger-Keldysh formalism, making use of the fully renormalized one-loop selfenergy computed in Ref. [27]. That computation is left for future work.

\section{Superhorizon hierarchies}

The final results for the superhorizon correlators in both Sec. 4 and 5 exhibit two types of hierarchies when in time-coincidence $\left(\eta^{\prime}=\eta\right)$. Firstly, the secular late-time 
hierarchy, where given enough time the hierarchy develops because of secular growth, and secondly, the spatial running hierarchy, where the larger the spatial separation, the larger the relative magnitude of correlators. For the conserved current correlators the hierarchy (up to numerical factors and tensor structures) takes the form,

$$
\begin{aligned}
\left\langle\hat{J}_{0}(\eta, \vec{x}) \hat{J}_{0}\left(\eta, \vec{x}^{\prime}\right)\right\rangle /\left\langle\hat{J}_{0}(\eta, \vec{x}) \hat{J}_{i}\left(\eta, \vec{x}^{\prime}\right)\right\rangle /\left\langle\hat{J}_{i}(\eta, \vec{x}) \hat{J}_{j}\left(\eta, \vec{x}^{\prime}\right)\right\rangle & \stackrel{\text { SH }}{\sim} 1 / \mathcal{H}\|\Delta \vec{x}\| /(\mathcal{H}\|\Delta \vec{x}\|)^{2},
\end{aligned}
$$

and for the E\&M correlators,

$$
\begin{aligned}
\left\langle\hat{E}_{i}(\eta, \vec{x}) \hat{E}_{j}\left(\eta, \vec{x}^{\prime}\right)\right\rangle /\left\langle\hat{E}_{i}(\eta, \vec{x}) \hat{B}_{j}\left(\eta, \vec{x}^{\prime}\right)\right\rangle /\left\langle\hat{B}_{i}(\eta, \vec{x}) \hat{B}_{j}\left(\eta, \vec{x}^{\prime}\right)\right\rangle & \stackrel{\mathrm{SH}}{\sim}(\mathcal{H}\|\Delta \vec{x}\|)^{2} / \mathcal{H}\|\Delta \vec{x}\| / 1 .
\end{aligned}
$$

These hierarchies seem very similar, as the ratios are powers of $(\mathcal{H}\|\Delta \vec{x}\|)$. However, they are actually independent of each other and have different origins. The current hierarchies are a consequence of the covariant conservation of the $U(1)$ current (4.2), while the E\&M hierarchies are a consequence of Faraday's law (5.7). In Sec. 4 and 5 these two laws given by homogeneous equations have been utilized as consistency checks of the solutions obtained from the inhomogeneous equations. Here we demonstrate that it is these laws that are responsible for the hierarchies in the first place.

The relevance of Faraday's law for redshifting of superhorizon magnetic fields was pointed out recently in [52]. There it was found to lead to very different scaling of magnetic fields in the radiation-dominated epoch, provided there was a large electric field generated in inflation. While in decelerating periods of expansion Faraday's law tells us something about the transients, in inflation it is responsible for establishing a definite hierarchy between the E\&M correlators, on the account of accelerated expansion of the background space-time. The conservation equation does the same for current correlators.

The hierarchies are not specific to the particular model we examined in this paper, but rather arise generically in inflation, and are expected to hold at the nonperturbative level as well. The expressions we give are specific to power-law inflation, but should get only small corrections in general slow-roll inflation. On the other hand, the amplitude of the correlators is not universal, and does depend on the model, etc. In the following Sec. 6.1 and 6.2 we give a brief account on the general structure of hierarchies between the current correlators, and between the E\&M correlators. The detailed study is left for future work.

\subsection{Conserved currents}

Provided that the covariant conservation of the currents is not broken by quantum effects, we may use the conservation equation (4.2) as an operator identity, and 
we may form 2-point functions (correlators) of conserved current operators. Let us assume that at superhorizon separations the (00) current correlator has the following leading asymptotic behaviour,

$$
\left\langle\hat{J}_{0}(x) \hat{J}_{0}\left(x^{\prime}\right)\right\rangle \stackrel{\overline{\mathrm{SH}}}{\sim} \mathcal{N}\|\Delta \vec{x}\|^{\alpha}\left(a a^{\prime}\right)^{\beta} .
$$

It follows immediately solely from the conservation equation that the remaining current correlators are,

$$
\begin{aligned}
\left\langle\hat{J}_{0}(x) \hat{J}_{i}\left(x^{\prime}\right)\right\rangle \stackrel{\mathrm{SH}}{\sim}\left\langle\hat{J}_{0}(x) \hat{J}_{0}\left(x^{\prime}\right)\right\rangle \times\left[-\left(\frac{2+\beta}{3+\alpha}\right)\left(\mathcal{H}^{\prime}\|\Delta \vec{x}\|\right)\right] \times \frac{\Delta x_{i}}{\|\Delta \vec{x}\|}, \\
\left\langle\hat{J}_{i}(x) \hat{J}_{j}\left(x^{\prime}\right)\right\rangle \stackrel{\overline{\mathrm{SH}}}{\sim}\left\langle\hat{J}_{0}(x) \hat{J}_{0}\left(x^{\prime}\right)\right\rangle \times\left[-\frac{(2+\beta)^{2}}{(2+\alpha)(3+\alpha)}\left(\mathcal{H} \mathcal{H}^{\prime}\|\Delta \vec{x}\|^{2}\right)\right] \\
\quad \times\left[\left(1+\frac{(3+\alpha)(4+\alpha)}{(2+\beta)^{2}} \#\right) \delta_{i j}-\frac{(2+\alpha)(3+\alpha)}{(2+\beta)^{2}} \# \frac{\Delta x_{i} \Delta x_{j}}{\|\Delta \vec{x}\|^{2}}\right],
\end{aligned}
$$

where \# is an undetermined constant. This is precisely the hierarchy observed in (4.9)-(4.11) that we have derived here without refering to the actual model, or making coupling constant expansions.

\subsection{E\&M fields}

Let us assume that the magnetic field correlator at superhorizon separations behaves at leading order as, ${ }^{10}$

$$
\left\langle\hat{B}_{i}(x) \hat{B}_{j}\left(x^{\prime}\right)\right\rangle \stackrel{\overline{\mathrm{SH}}}{\sim} \mathcal{N}\|\Delta \vec{x}\|^{\alpha}\left(a a^{\prime}\right)^{\beta} \times\left[(\alpha+2) \delta_{i j}-\alpha \frac{\Delta x_{i} \Delta x_{j}}{\|\Delta \vec{x}\|^{2}}\right],
$$

such that it satisfies the condition of no magnetic charge from (5.7). Then the remaining E\&M correlators follow from the Faraday's law in (5.7),

$$
\begin{aligned}
\left\langle\hat{E}_{i}(x) \hat{B}_{j}\left(x^{\prime}\right)\right\rangle \stackrel{\overline{\mathrm{SH}}}{\sim} \mathcal{N}\left(a a^{\prime}\right)^{\beta}\|\Delta \vec{x}\|^{\alpha} \times[-\beta(\mathcal{H}\|\Delta \vec{x}\|)] \times \varepsilon_{i j k} \frac{\Delta x_{k}}{\|\Delta \vec{x}\|}, \\
\left\langle\hat{E}_{i}(x) \hat{E}_{j}\left(x^{\prime}\right)\right\rangle \stackrel{\overline{\mathrm{SH}}}{\sim} \mathcal{N}\left(a a^{\prime}\right)^{\beta}\|\Delta \vec{x}\|^{\alpha} \times\left[-\beta\left(\mathcal{H} \mathcal{H}^{\prime}\|\Delta \vec{x}\|^{2}\right)\right] \\
\times\left[\# \delta_{i j}-\left(\beta^{2}-(2+\alpha) \#\right) \frac{\Delta x_{i} \Delta x_{j}}{\|\Delta \vec{x}\|^{2}}\right],
\end{aligned}
$$

where \# is an undetermined constant. Thus, Faraday's law precisely leads to the superhorizon hierarchies observed in (5.27)-(5.29), which we have derived without assuming a particular model, or any particular approximation scheme, Therefore.

\footnotetext{
${ }^{10}$ Note that this is not the most general scaling that can result in inflation, since we had already found that in the de Sitter limit (5.35)-(5.37) the power-law behaviour turns into logarithmic one. However, generalizations to include these cases are straightforward, and we do not consider such details here.
} 
this is a general feature of E\&M correlators at superhorizon separations in inflation. Strictly speaking, the hierarchy in this section has been derived for power-law infation, but we expect the results to hold with small corrections in general power-law inflation. Recently the tree-level E\&M of the Abelian Higgs model have been worked out in power-law inflation [48], and they exactly respect the super-Hubble hierarchy in $(6.2)$.

\section{Discussion}

In this work we have examined how the vector in the massless SQED model responds to the charged current fluctuations of the complex scalar induced by the accelerating expansion of power-law inflation. More precisely, the effects that we have taken into account are

- The creation of $U(1)$ charge fluctuations of the complex scalar due to the expansion,

- Reaction of the $U(1)$ vector field to the gravitationally created charge fluctuations of the complex scalar,

which constitute one-loop effects. The effect is quantified by the two-point functions of conserved currents (4.9)-(4.11), which source the two-point function of electric and magnetic field fluctuations, given in (5.27)-(5.29); these constitute the main result of the paper. The E\&M correlators exhibit three relevant properties,

- Secular enhancement - growth in time compared to the tree-level correlators

- Spatial running - growth with spatial separation compared to the tree-level correlators,

- IR sensitivity - dependence on the IR scale present in the scalar propagator.

All three point to potentially large non-perturbative IR effects. One might wonder whether the secular enhancement and spatial running go hand-in-hand with IR sensitivity, and would be removed by resumming the leading orders at each loop. This, however, does not seem to be the case, since all the E\&M correlators have the same dependence on the IR scale $k_{0}$, but different secular and spatial running.

It is noteworthy that both the components of the tree-level conserved current correlators (4.9)-(4.11), and the one-loop E\&M correlators (5.27)-(5.29) satisfy hierarchies, (6.1) and (6.2), respectively. Even though our explicit results pertain to the one-loop level, the hierarchies correlators satisfy must hold at the non-perturbative level as well, as they are consequences of operator versions of current conservation and Faraday's law. They have recently been confirmed for the Abelian Higgs model in power-law inflation [48]. 
The results presented here open up several directions for further investigation. The large electric field fluctuations coherent over superhorizon separations might dynamically provide the electric field necessary for the occurrence of the Schwinger mechanism - production of charged pairs in external electric fields - that has attracted attention recently [61-63], more so due to possible imprints it might leave on the cosmological observables. Furthermore, technical simplifications brought about by casting the problem in terms of double-differential equations (5.10)-(5.12) for correlators make two-loop computation far more feasible. These equations can be readily solved in the superhorizon limit, and eliminate the need of computing some of the integrals associated with Feynman diagrams. At one-loop there was no need for regularization and renormalization since we examined off-coincident full twopoint functions. At two-loop order we likely require only renormalization of the one-loop vacuum polarization and scalar self-mass in power-law inflation; both are eminently feasible computations that have thus far been performed only in de Sitter space $[27,31,36]$.

Finally, the properties of the correlators discussed in this work make a good case for the eventual breakdown of perturbation theory. The question then arises on whether this breakdown signifies the existence of large non-perturbative effects. This question can only be answered by going beyond perturbation theory in the IR, for example by setting up the full set of corresponding Starobinsky's stochastic equations, generalizing the approach of $[42,64]$, and examining both the vector and complex scalar. The Renormalization Group can then be used in this stochastic context to examine properties of the correlators at large spatial separations, see [65] for an application to a test field in de Sitter. This will require adaptation of existing techniques. Furthermore, obtaining further perturbative results to higher loop orders, using the techniques discussed in this work, would be very useful as (i) they would establish how higher order physical processes scale in time and with spatial separation, and (ii) would provide a consistency check that any resummation method has to reproduce. We hope to return to all these issues in future work.

\section{Acknowledgments}

D. G. would like to thank Takeshi Kobayashi for the discussions on the subject during the visit to ICTP in Trieste. This work was partially supported by the Fonds de la Recherche Scientifique - FNRS under Grant IISN 4.4517.08 - Theory of fundamental interactions, and by the STFC grant ST/P000371/1 - Particles, Fields and Spacetime.

\section{References}

[1] N. Arkani-Hamed and J. Maldacena, Cosmological Collider Physics, 1503.08043. 
[2] P.D. Meerburg, M. Münchmeyer, J.B. Muñoz and X. Chen, Prospects for Cosmological Collider Physics, JCAP 03 (2017) 050 [1610.06559].

[3] L. Parker, Particle creation in expanding universes, Phys. Rev. Lett. 21 (1968) 562.

[4] L. Parker, Quantized fields and particle creation in expanding universes. 1., Phys. Rev. 183 (1969) 1057.

[5] N.D. Birrell and P.C.W. Davies, Quantum Fields in Curved Space, Cambridge University Press, Cambridge, UK (1984), 10.1017/CBO9780511622632.

[6] V. Mukhanov and S. Winitzki, Introduction to Quantum Effects in Gravity, Cambridge University Press, Cambridge, UK (2007), 10.1017/CBO9780511809149.

[7] L.E. Parker and D. Toms, Quantum Field Theory in Curved Spacetime: Quantized Field and Gravity, Cambridge University Press, Cambridge, UK (2009), 10.1017/CBO9780511813924.

[8] L.H. Ford and A. Vilenkin, Global Symmetry Breaking in Two-dimensional Flat Space-time and in De Sitter Space-time, Phys. Rev. D 33 (1986) 2833.

[9] A.A. Starobinsky and J. Yokoyama, Equilibrium state of a selfinteracting scalar field in the De Sitter background, Phys. Rev. D 50 (1994) 6357 [astro-ph/9407016].

[10] G. Lazzari and T. Prokopec, Symmetry breaking in de Sitter: a stochastic effective theory approach, 1304.0404.

[11] J. Serreau, Renormalization group flow and symmetry restoration in de Sitter space, Phys. Lett. B 730 (2014) 271 [1306.3846].

[12] M. Guilleux and J. Serreau, Quantum scalar fields in de Sitter space from the nonperturbative renormalization group, Phys. Rev. D 92 (2015) 084010 [1506.06183].

[13] A.D. Dolgov, Conformal Anomaly and the Production of Massless Particles by a Conformally Flat Metric, Sov. Phys. JETP 54 (1981) 223.

[14] A. Benevides, A. Dabholkar and T. Kobayashi, To B or not to B: Primordial magnetic fields from Weyl anomaly, JHEP 11 (2018) 039 [1808.08237].

[15] X. Chen, Y. Wang and Z.-Z. Xianyu, Loop Corrections to Standard Model Fields in Inflation, JHEP 08 (2016) 051 [1604.07841].

[16] X. Chen, Y. Wang and Z.-Z. Xianyu, Standard Model Background of the Cosmological Collider, Phys. Rev. Lett. 118 (2017) 261302 [1610.06597].

[17] X. Chen, Y. Wang and Z.-Z. Xianyu, Standard Model Mass Spectrum in Inflationary Universe, JHEP 04 (2017) 058 [1612.08122].

[18] A. Maleknejad, M.M. Sheikh-Jabbari and J. Soda, Gauge Fields and Inflation, Phys. Rept. 528 (2013) 161 [1212.2921].

[19] R. Durrer and A. Neronov, Cosmological Magnetic Fields: Their Generation, Evolution and Observation, Astron. Astrophys. Rev. 21 (2013) 62 [1303.7121]. 
[20] K. Subramanian, The origin, evolution and signatures of primordial magnetic fields, Rept. Prog. Phys. 79 (2016) 076901 [1504.02311].

[21] M.S. Turner and L.M. Widrow, Inflation Produced, Large Scale Magnetic Fields, Phys. Rev. D 37 (1988) 2743.

[22] M. Giovannini and M.E. Shaposhnikov, Primordial magnetic fields from inflation?, Phys. Rev. D 62 (2000) 103512 [hep-ph/0004269].

[23] E.A. Calzetta, A. Kandus and F.D. Mazzitelli, Primordial magnetic fields induced by cosmological particle creation, Phys. Rev. D 57 (1998) 7139 [astro-ph/9707220].

[24] A. Kandus, E.A. Calzetta, F.D. Mazzitelli and C.E.M. Wagner, Cosmological magnetic fields from gauge mediated supersymmetry breaking models, Phys. Lett. B 472 (2000) 287 [hep-ph/9908524].

[25] A.-C. Davis, K. Dimopoulos, T. Prokopec and O. Tornkvist, Primordial spectrum of gauge fields from inflation, Phys. Lett. B 501 (2001) 165 [astro-ph/0007214].

[26] K. Dimopoulos, T. Prokopec, O. Tornkvist and A.C. Davis, Natural magnetogenesis from inflation, Phys. Rev. D 65 (2002) 063505 [astro-ph/0108093].

[27] T. Prokopec, O. Tornkvist and R.P. Woodard, One loop vacuum polarization in a locally de Sitter background, Annals Phys. 303 (2003) 251 [gr-qc/0205130].

[28] T. Prokopec, O. Tornkvist and R.P. Woodard, Photon mass from inflation, Phys. Rev. Lett. 89 (2002) 101301 [astro-ph/0205331].

[29] T. Prokopec and R.P. Woodard, Vacuum polarization and photon mass in inflation, Am. J. Phys. 72 (2004) 60 [astro-ph/0303358].

[30] T. Prokopec and R.P. Woodard, Dynamics of superhorizon photons during inflation with vacuum polarization, Annals Phys. 312 (2004) 1 [gr-qc/0310056].

[31] T. Prokopec and E. Puchwein, Photon mass generation during inflation: de Sitter invariant case, JCAP 04 (2004) 007 [astro-ph/0312274].

[32] T. Prokopec and E. Puchwein, Nearly minimal magnetogenesis, Phys. Rev. D 70 (2004) 043004 [astro-ph/0403335].

[33] F.K. Popov, Debye mass in de Sitter space, JHEP 06 (2018) 033 [1711.11010].

[34] T. Prokopec, N.C. Tsamis and R.P. Woodard, Stochastic Inflationary Scalar Electrodynamics, Annals Phys. 323 (2008) 1324 [0707.0847].

[35] A.A. Starobinsky, STOCHASTIC DE SITTER (INFLATIONARY) STAGE IN THE EARLY UNIVERSE, Lect. Notes Phys. 246 (1986) 107.

[36] E.O. Kahya and R.P. Woodard, Charged scalar self-mass during inflation, Phys. Rev. D 72 (2005) 104001 [gr-qc/0508015].

[37] E.O. Kahya and R.P. Woodard, One Loop Corrected Mode Functions for SQED during Inflation, Phys. Rev. D 74 (2006) 084012 [gr-qc/0608049]. 
[38] T. Prokopec, N.C. Tsamis and R.P. Woodard, Two Loop Scalar Bilinears for Inflationary SQED, Class. Quant. Grav. 24 (2007) 201 [gr-qc/0607094].

[39] T. Prokopec, N.C. Tsamis and R.P. Woodard, Two loop stress-energy tensor for inflationary scalar electrodynamics, Phys. Rev. D 78 (2008) 043523 [0802.3673].

[40] L.-Y. Chen, N. Goldenfeld and Y. Oono, The Renormalization group and singular perturbations: Multiple scales, boundary layers and reductive perturbation theory, Phys. Rev. E 54 (1996) 376 [hep-th/9506161].

[41] C.P. Burgess, L. Leblond, R. Holman and S. Shandera, Super-Hubble de Sitter Fluctuations and the Dynamical RG, JCAP 03 (2010) 033 [0912.1608].

[42] N.C. Tsamis and R.P. Woodard, Stochastic quantum gravitational inflation, Nucl. Phys. B 724 (2005) 295 [gr-qc/0505115].

[43] R.P. Woodard, A Leading logarithm approximation for inflationary quantum field theory, Nucl. Phys. B Proc. Suppl. 148 (2005) 108 [astro-ph/0502556].

[44] B. Garbrecht, G. Rigopoulos and Y. Zhu, Infrared correlations in de Sitter space: Field theoretic versus stochastic approach, Phys. Rev. D 89 (2014) 063506 [1310.0367].

[45] B. Garbrecht, F. Gautier, G. Rigopoulos and Y. Zhu, Feynman Diagrams for Stochastic Inflation and Quantum Field Theory in de Sitter Space, Phys. Rev. D 91 (2015) 063520 [1412.4893].

[46] D. López Nacir, F.D. Mazzitelli and L.G. Trombetta, $O(N)$ model in Euclidean de Sitter space: beyond the leading infrared approximation, JHEP 09 (2016) 117 [1606.03481].

[47] T.M. Janssen, S.P. Miao, T. Prokopec and R.P. Woodard, The Hubble Effective Potential, JCAP 05 (2009) 003 [0904.1151].

[48] D. Glavan, A. Marunović, T. Prokopec and Z. Zahraee, Abelian Higgs model in power-law inflation: the propagators in the unitary gauge, JHEP 09 (2020) 165 [2005. 05435].

[49] J. Berges, Introduction to nonequilibrium quantum field theory, AIP Conf. Proc. 739 (2004) 3 [hep-ph/0409233].

[50] D. Glavan and T. Prokopec, A pedestrian introduction to non-equilibrium QFT, https://webspace.science.uu.nl/ proko101/LecturenotesNonEquilQFT.pdf .

[51] A. Kaya, Superhorizon Electromagnetic Field Background from Higgs Loops in Inflation, JCAP 03 (2018) 046 [1801.02032].

[52] T. Kobayashi and M.S. Sloth, Early Cosmological Evolution of Primordial Electromagnetic Fields, Phys. Rev. D 100 (2019) 023524 [1903.02561].

[53] M. D'Onofrio, R.N. Lerner and A. Rajantie, Electrically charged curvaton, JCAP 10 (2012) 004 [1207.1063]. 
[54] C. Goolsby-Cole and L. Sorbo, On the electric charge of the observable Universe, 1511.07465.

[55] T.M. Janssen, S.P. Miao, T. Prokopec and R.P. Woodard, Infrared Propagator Corrections for Constant Deceleration, Class. Quant. Grav. 25 (2008) 245013 [0808.2449].

[56] N.A. Chernikov and E.A. Tagirov, Quantum theory of scalar fields in de Sitter space-time, Ann. Inst. H. Poincare Phys. Theor. A 9 (1968) 109.

[57] T.S. Bunch and P.C.W. Davies, Quantum Field Theory in de Sitter Space: Renormalization by Point Splitting, Proc. Roy. Soc. Lond. A 360 (1978) 117.

[58] L.H. Ford and L. Parker, Infrared Divergences in a Class of Robertson-Walker Universes, Phys. Rev. D 16 (1977) 245.

[59] T.M. Janssen and T. Prokopec, Regulating the infrared by mode matching: A Massless scalar in expanding spaces with constant deceleration, Phys. Rev. D 83 (2011) 084035 [0906.0666].

[60] K. Lochan, K. Rajeev, A. Vikram and T. Padmanabhan, Quantum correlators in Friedmann spacetimes: The omnipresent de Sitter spacetime and the invariant vacuum noise, Phys. Rev. D 98 (2018) 105015 [1805.08800].

[61] T. Kobayashi and N. Afshordi, Schwinger Effect in $4 D$ de Sitter Space and Constraints on Magnetogenesis in the Early Universe, JHEP 10 (2014) 166 [1408.4141].

[62] M.B. Fröb, J. Garriga, S. Kanno, M. Sasaki, J. Soda, T. Tanaka et al., Schwinger effect in de Sitter space, JCAP 04 (2014) 009 [1401.4137].

[63] M. Banyeres, G. Domènech and J. Garriga, Vacuum birefringence and the Schwinger effect in $(3+1)$ de Sitter, JCAP 10 (2018) 023 [1809.08977].

[64] I. Moss and G. Rigopoulos, Effective long wavelength scalar dynamics in de Sitter, JCAP 05 (2017) 009 [1611.07589].

[65] T. Prokopec and G. Rigopoulos, Functional renormalization group for stochastic inflation, JCAP 08 (2018) 013 [1710.07333]. 